\title{
An Energy- and Charge-conserving, Implicit, Electrostatic Particle-in-Cell Algorithm
}

\author{
G. Chen, L. Chacón, \\ Oak Ridge National Laboratory, Oak Ridge,TN 37831, USA \\ D. C. Barnes \\ Coronado Consulting, Lamy, NM 87540, USA
}

\begin{abstract}
This paper discusses a novel fully implicit formulation for a one-dimensional electrostatic particle-in-cell (PIC) plasma simulation approach. Unlike earlier implicit electrostatic PIC approaches (which are based on a linearized Vlasov-Poisson formulation), ours is based on a nonlinearly converged Vlasov-Ampère (VA) model. By iterating particles and fields to a tight nonlinear convergence tolerance, the approach features superior stability and accuracy properties, avoiding most of the accuracy pitfalls in earlier implicit PIC implementations. In particular, the formulation is stable against temporal (Courant-Friedrichs-Lewy) and spatial (aliasing) instabilities. It is charge- and energy-conserving to numerical round-off for arbitrary implicit time steps (unlike the earlier "energy-conserving" explicit PIC formulation, which only conserves energy in the limit of arbitrarily small time steps). While momentum is not exactly conserved, errors are kept small by an adaptive particle sub-stepping orbit integrator, which is instrumental to prevent particle tunneling (a deleterious effect for long-term accuracy). The VA model is orbit-averaged along particle orbits to enforce an energy conservation theorem with particle sub-stepping. As a result, very large time steps, constrained only by the dynamical time scale of interest, are possible without accuracy loss. Algorithmically, the approach features a Jacobian-free Newton-Krylov solver. A main development in this study is the nonlinear elimination of the new-time particle variables (positions and velocities). Such nonlinear elimination, which we term particle enslavement, results in a nonlinear formulation with memory requirements comparable to those of a fluid computation, and affords us substantial freedom in regards to the particle orbit integrator. Numerical examples are presented that demonstrate the advertised properties of the scheme. In particular, long-time ion acoustic wave simulations show that numerical accuracy does not degrade even with very large implicit time steps, and that significant CPU gains are possible.
\end{abstract}

\section{Introduction}

The evolution of collisionless plasmas in the presence of electromagnetic fields is well described by the Vlasov-Maxwell set of equations. The Vlasov equation describes the evolution of the probability distribution functions (PDFs) for one or more species, while electromagnetic fields evolve according to Maxwell's equations. The field-PDF description is tightly 
coupled. Maxwell's equations (or a subset thereof) are driven by moments of the PDF such as charge density and/or current density. The PDF, on the other hand, follows a hyperbolic equation in phase space, whose characteristics are self-consistently determined by the fields.

The numerical solution of such strongly coupled systems has proved challenging. Here, we concern ourselves with particle-in-cell (PIC) techniques [1-3], which integrate the VlasovMaxwell equations in time by combining a mesh (to represent fields) with particles (to follow characteristics of the PDF in phase space). The PIC approach has been very successful, enabling many first-principle kinetic calculations of plasma dynamics since the early years of computer simulations (e.g. [4]). Key aspects of the method involve the definition of interpolation operations between mesh and particle quantities, and the coupled temporal integration of field and particle equations.

The most common PIC implementation employs an explicit time integration of the field and particle equations [1] (e.g., the full- $f$ explicit momentum-conserving method). Explicit approaches provide a straightforward recipe for temporal integration. However, standard explicit PIC approaches suffer from temporal numerical stability constraints (the well-known Courant-Friedrichs-Lewy or CFL time step limit). Furthermore, some explicit PIC implementations also feature spatial stability constraints (e.g., the momentum-conserving explicit Vlasov-Poisson PIC formulation, which requires the Debye length to be resolved in order to avoid so-called finite-grid instabilities) [1. As a result of these stability constraints, explicit PIC becomes very demanding computationally when applied to multidimensional configurations in the presence of general electromagnetic fields[5].

Implicit methods, however, can free the PIC approach from numerical stability constraints, and thus have the potential of much improved algorithmic efficiency. This realization drove the exploration of implicit PIC starting in the 1980s [6-20]. These studies explored the viability of an implicit PIC formulation and its accuracy properties, and resulted in important developments such as the implicit-moment method [6, 9, 14, 20] and the direct-implicit method [10, 12, 15, 17, 19]. However, limitations of the solver technology at the time forced early implicit PIC practitioners to rely on approximations such as linearization and lagging, which did not respect the strong field-particle coupling. These numerical approximations produced energy conservation errors that could result in significant plasma self-heating or self-cooling [21].

In this study, we explore a fully implicit PIC solver, based on Newton-Krylov methods [22], in which field-particle couplings are converged to a tight nonlinear tolerance. For this proof-of-principle study, we employ a one-dimensional (1D) electrostatic Vlasov-Ampère (VA) model. This study builds on earlier studies for the 1D Vlasov-Poisson (VP) [23] and VA 24] equations. The emphasis here is on both accuracy and efficiency. By iterating particles and fields to a tight convergence tolerance, the approach features superior stability and accuracy properties, avoiding most of the accuracy pitfalls in earlier implicit PIC implementations. In particular, we will show that the formulation is stable against both temporal and spatial instabilities. The formulation is charge- and energy-conserving to numerical roundoff for arbitrary implicit time steps. This is unlike the explicit "energy-conserving" PIC formulation of Lewis [25], which conserves energy only in the limit of arbitrarily small time steps. Automatic exact charge conservation[26] is achieved by ensuring that each particle moves within a given cell. Momentum is not exactly conserved in our approach, but errors are kept small by consistent spatial smoothing and an adaptive particle sub-stepping orbit 
integrator, which is instrumental to prevent particle tunneling (a deleterious effect for longterm accuracy). The time-centered VA equations are orbit-averaged along the sub-stepped particle orbits [27] to enable an exact energy conservation theorem. As a result, very large time steps, constrained only by the collective dynamical time scale of interest, are possible for the field solver without significant accuracy loss.

Algorithmically, the approach features an unpreconditioned Jacobian-free Newton-Krylov (JFNK) solver. A main algorithmic contribution of this study is the nonlinear elimination of the new-time particle variables (positions and velocities), which we term particle enslavement. This results in a two-fold advantage. On one hand, the nonlinear formulation features computer memory requirements comparable to those of a fluid computation. On the other hand, it affords us substantial freedom in regards to the particle orbit integrator, enabling the implementation of an adaptive charge-conserving mover, as described earlier.

Numerical examples are presented that demonstrate the advertised properties of the scheme. In particular, we have performed multiscale ion acoustic wave (IAW) simulations. The numerical results show that an accurate orbit integration is key for long-term accuracy. Furthermore, our results demonstrate that a standard explicit VP momentum-conserving PIC solver requires a timestep much smaller than the explicit CFL to provide comparable accuracy to the fully implicit PIC algorithm employing a much larger time step. Efficiencywise, we argue that large CPU gains are possible, especially in multiple dimensions, when the system size is much larger than the Debye length. We demonstrate moderate CPU gains numerically with the 1D IAW problem with our unpreconditioned Newton-Krylov solver, underscoring the algorithmic potential of the approach.

The rest of the paper is organized as follows. Section 2 describes the Vlasov-Ampère model in the continuum and its application to 1D electrostatic plasma simulation. Section 3 describes the details of our discrete Vlasov-Ampère implementation, with emphasis on energy and charge conservation, as well as our adaptive particle mover. Section 4 describes the concept of particle enslavement, and provides details of our JFNK solver implementation. Section 5 demonstrates the advertised accuracy and efficiency properties of the scheme with standard electrostatic problems such as Landau damping, two-stream instability, and the ion acoustic wave. Finally, we conclude in section 6 .

\section{Electrostatic Vlasov-Ampère model}

A collisionless electrostatic plasma is described by the Vlasov-Poisson equations:

$$
\begin{aligned}
\frac{\partial f_{\alpha}}{\partial t}+\mathbf{v} \cdot \nabla f_{\alpha}+\frac{q_{\alpha}}{m_{\alpha}} \mathbf{E} \cdot \nabla_{v} f_{\alpha} & =0 \\
\nabla \cdot \mathbf{E} & =\frac{\rho}{\epsilon_{0}}, \\
\mathbf{E} & =-\nabla \phi,
\end{aligned}
$$

where $f_{\alpha}(\mathbf{r}, \mathbf{v})$ is the particle distribution function of species $\alpha$ in phase space, $q_{\alpha}$ and $m_{\alpha}$ are the species charge and mass respectively, $\phi$ and $\mathbf{E}$ are the self-consistent electric potential and field respectively, $\rho(\mathbf{r})=\sum_{\alpha} q_{\alpha} \int d \mathbf{v} f_{\alpha}(\mathbf{r}, \mathbf{v})$ is the charge density, and $\epsilon_{0}$ is the vacuum permittivity. This system of equations is commonly used for modeling the behavior of an electrostatic plasma in one or more dimensions [1. An alternate formulation can be written 
using Ampère's law, which is derived in the electrostatic limit as follows. We start with the charge continuity equation:

$$
\frac{\partial \rho}{\partial t}+\nabla \cdot \mathbf{j}=0
$$

where $\mathbf{j}=\sum_{\alpha} q_{\alpha} \int \mathbf{v} d \mathbf{v} f_{\alpha}$ is the plasma current density. We substitute Eq.(2) in Eq.(4) to have

$$
\nabla \cdot\left(\epsilon_{0} \frac{\partial \mathbf{E}}{\partial t}+\mathbf{j}\right)=0
$$

In $1 \mathrm{D}$, one finds the integral of Eq.(5) exactly as

$$
\epsilon_{0} \frac{\partial E}{\partial t}+j=C(t)
$$

where $C(t)$ is an arbitrary function of time. By integrating Eq. 6) over the periodic domain, one finds $C(t)=\langle j\rangle$, where $\langle j\rangle=\int j d x / \int d x$ is the spatial average of the current density, and hence Eq. (6) becomes:

$$
\epsilon_{0} \frac{\partial E}{\partial t}+j=\langle j\rangle
$$

The right hand side of Eq. (7) is a solvability condition for the $1 \mathrm{D}$ electrostatic Ampère equation. It is also required to preserve Galilean invariance of the system.

In the continuum, the VP and VA formulations are equivalent. In the discrete, however, they have different properties. Generally, VP is momentum and charge conserving, while VA can be energy and charge conserving, as we will show. We will also show that the discrete VA and VP can be equivalent under special conditions [see Sec.(3.6)]. In what follows, we consider the VA formulation, as it generally has better stability properties [27.

The VA model can be extended to multi-dimensions by replacing $j-\langle j\rangle$ with the longitudinal piece of the current, $\mathbf{j}+\nabla \times\left(\nabla^{-2}(\nabla \times \mathbf{j})\right.$, where $\nabla^{-2}$ is the inverse Laplace operator. The latter expression ensures that the electric field obtained from Ampère's law is conservative [i.e., that Eq. (3) is enforced]. Alternatively, one can derive an evolution equation for the electric field (or, equivalently, the electrostatic potential $\phi$ ) by using the charge continuity equation Eq.(4), Gauss' law Eq.(2), and the electrostatic approximation Eq.(3) to find:

$$
\epsilon_{0} \frac{\partial \nabla^{2} \phi}{\partial t}-\nabla \cdot \mathbf{j}=0
$$

with $\mathbf{E}$ found from Eq.(3). This equation also requires inverting a Laplace operator to find $\phi$.

\section{Implicit particle-based discretization of the VA model}

We begin to develop a suitable implicit discretization of the VA equations by considering a time-centered [Crank-Nicolson $(\mathrm{CN})]$ formulation, written as

$$
\begin{aligned}
\epsilon_{0} \frac{E_{i}^{n+1}-E_{i}^{n}}{\Delta t}+j_{i}^{n+1 / 2} & =\langle j\rangle^{n+1 / 2}, \\
\frac{x_{p}^{n+1}-x_{p}^{n}}{\Delta t} & =v_{p}^{n+1 / 2}, \\
\frac{v_{p}^{n+1}-v_{p}^{n}}{\Delta t} & =\frac{q_{p}}{m_{p}} E\left(x_{p}^{n+1 / 2}\right),
\end{aligned}
$$


where subscripts $i$ and $p$ denote grid points and particles respectively, superscript $n$ denotes time step, $j_{i}^{n+1 / 2}=\sum_{p} q_{p} S\left(x_{i}-x_{p}^{n+1 / 2}\right) v_{p}^{n+1 / 2} / \Delta x$ is the time-centered plasma current density, $E\left(x_{p}^{n+1 / 2}\right)=\sum_{i} S\left(x_{i}-x_{p}^{n+1 / 2}\right)\left(E_{i}^{n+1}+E_{i}^{n}\right) / 2$ is the time-centered electric field, and the half time step particle position and velocity are defined by $x_{p}^{n+1 / 2}=\left(x_{p}^{n}+x_{p}^{n+1}\right) / 2$ and $v_{p}^{n+1 / 2}=\left(v_{p}^{n}+v_{p}^{n+1}\right) / 2$, respectively. The CN method is implicit (a particle's future position and velocity depend on the future acceleration), symplectic, second order accurate in time, and unconditionally stable. Most importantly, CN is non-dissipative, which enables an exact energy conservation theorem. Next, we proceed to show that the time centered VA equations (8)-(10) admit a discrete energy conservation theorem.

\subsection{Exact energy conservation theorem}

Multiplying Eq. 10 by $v_{p}^{n+1 / 2}$ and summing over all particles, we find that

$$
\begin{aligned}
\sum_{p} \frac{1}{2} m_{p}\left(v_{p}^{n+1}+v_{p}^{n}\right)\left(v_{p}^{n+1}-v_{p}^{n}\right) & =\sum_{p} v_{p}^{n+1 / 2} q_{p} E\left(x_{p}^{n+1 / 2}\right) \Delta t \\
\text { introducing def. of } E\left(x_{p}^{n+1 / 2}\right) \rightarrow & =\sum_{p} \Delta t v_{p}^{n+1 / 2} q_{p} \sum_{i} \frac{E_{i}^{n+1}+E_{i}^{n}}{2} S\left(x_{i}-x_{p}^{n+1 / 2}\right) \\
\text { commuting sums } \rightarrow & =\sum_{i} \Delta t j_{i}^{n+1 / 2} \frac{E_{i}^{n+1}+E_{i}^{n}}{2} \Delta x \\
\text { using Ampère's law } \rightarrow & =\sum_{i}\left[\Delta t\langle j\rangle^{n+1 / 2}-\epsilon_{0}\left(E_{i}^{n+1}-E_{i}^{n}\right)\right] \frac{E_{i}^{n+1}+E_{i}^{n}}{2} \Delta x \\
\sum_{i} E_{i}^{n(+1)}=0 & =-\sum_{i} \frac{\epsilon_{0}}{2}\left[\left(E_{i}^{n+1}\right)^{2}-\left(E_{i}^{n}\right)^{2}\right] \Delta x
\end{aligned}
$$

where the last step is justified because $E$ is a gradient of $\phi$ and we are considering a periodic domain. We therefore conclude that

$$
\left[\sum_{p} \frac{1}{2} m_{p}\left(v_{p}\right)^{2}+\sum_{i} \frac{\Delta x}{2} \epsilon_{0}\left(E_{i}\right)^{2}\right]^{n+1}=\left[\sum_{p} \frac{1}{2} m_{p}\left(v_{p}\right)^{2}+\sum_{i} \frac{\Delta x}{2} \epsilon_{0}\left(E_{i}\right)^{2}\right]^{n}
$$

i.e., the electrostatic system (8)-(10) features an exact energy conservation theorem, whereby the total energy [defined as the sum of kinetic energy $\left(\sum_{p} \frac{1}{2} m v_{p}^{2}\right)$ and the electric field energy $\left.\left(\sum_{i} \frac{\Delta x}{2} \epsilon_{0} E_{i}^{2}\right)\right]$ is exactly conserved from time step $n$ to time step $n+1$. We emphasize that this result is enabled by a VA formulation that uses (a) a CN time discretization and (b) identical current assignment and force interpolation shape functions. It is important to note that exact conservation of energy requires the implicit field and particle equations to be updated in a nonlinearly consistent manner every time step. This is distinctly different from previous implicit schemes [16, 28, 29], which do not feature nonlinear consistency. It is also different from the "energy-conserving" scheme developed by Lewis [25], which is explicit and does not conserves energy exactly with finite $\Delta t$.

The benefit of the existence of an energy conservation theorem is that finite-grid instabilities, commonly seen in explicit PIC simulations when $\Delta x>\lambda_{D}$, are eliminated. This 
property has the potential of significant computational savings of implicit PIC vs. explicit PIC, as many fewer grid points (and consequently fewer particles) will be required for the former in regimes where $k \lambda_{D} \ll 1$. Additionally, time steps much larger than $\Delta t_{C F L} \sim \omega_{p e}^{-1}$ are possible without numerical instabilities or numerical heating or cooling. However, stability is not the only requirement, as accuracy also needs to be ensured, particularly when employing large time steps. In the next section, we discuss the issue of accuracy in our implicit PIC implementation, and our approach to mitigate potential numerical errors.

\subsection{Particle sub-stepping and orbit-averaging}

In multiple time-scale problems, $\omega \ll \omega_{p e}$, and a large time step is desired. However, as pointed out by Langdon [28, 30], large inaccuracies in particle orbits may result when a large time step is employed, such that a particle moves a distance much greater than a cell size. The accuracy impact of orbit errors can be clearly seen by computing the dielectric constant of a homogeneous plasma [30]. For instance, in the intermediate frequency regime $\omega_{p i} \ll \omega \ll \omega_{p e}$, the electron response in the dielectric constant is expected from theory to be

$$
\epsilon_{\text {theory }} \cong 1+\left(k \lambda_{D}\right)^{-2}+(\text { ion response }) .
$$

With a $\mathrm{CN}$ discretization of particle orbits, however, the numerical response when $k v_{t h} \Delta t \gg$ 1 is

$$
\epsilon_{\mathrm{CN}} \cong 1+\frac{1}{4}\left(\omega_{p e} \Delta t\right)^{2}+(\text { ion response }),
$$

which is much larger than $\epsilon_{\text {theory }}$ in the limit considered. Thus, accuracy requires that

$$
\Delta t<\frac{\Delta x}{v_{t h}} .
$$

This is an undesirable constraint, especially for multiscale problems for which the time-scale of the slow dynamics is much larger than that determined by Eq.(12).

In this study, we address the issue of orbit accuracy by considering particle sub-stepping [27], where different time steps for orbit and field integration are employed: small steps $(\Delta \tau$ such that $k_{\max } v_{t h} \Delta \tau<1$ with $\left.k_{\max }=\pi / \Delta x\right)$ are used to ensure the accuracy of the particle orbits and the collective response; meanwhile, a larger time step $(\Delta t \geq \Delta \tau)$ is used in the field solver to follow the low-frequency dynamics. This approach allows slow particles to be integrated quickly and fast particles to be integrated accurately. We will discuss the numerical implementation of particle sub-stepping in the overall algorithm in Sec.(4).

In principle, particle sub-stepping breaks the energy conservation theorem. However, energy conservation can be recovered by considering an appropriate orbit-averaged plasma current density to advance $E$ in Eq.(8). The appropriate time-centered, orbit averaged current density is defined as

$$
\bar{j}_{i}^{n+1 / 2}=\frac{1}{\Delta x \Delta t} \sum_{\nu=1}^{N_{\nu}} \sum_{p} q_{p} S\left(x_{i}-x_{p}^{\nu+1 / 2}\right) v_{p}^{\nu+1 / 2} \Delta \tau^{\nu},
$$

where $\nu$ is the a sub-step index, $N_{\nu}$ is the number of sub-steps, and $\Delta t=\sum_{\nu=1}^{N_{\nu}} \Delta \tau^{\nu}$. Each sub-step is a CN move with time step $\Delta \tau^{\nu}$, which can change from one sub-step to another:

$$
\frac{x_{p}^{\nu+1}-x_{p}^{\nu}}{\Delta \tau^{\nu}}=v_{p}^{\nu+1 / 2}
$$




$$
\frac{v_{p}^{\nu+1}-v_{p}^{\nu}}{\Delta \tau^{\nu}}=\frac{q_{p}}{m_{p}} E\left(x_{p}^{\nu+1 / 2}\right),
$$

with $E\left(x_{p}^{\nu+1 / 2}\right)=\sum_{i} S\left(x_{i}-x_{p}^{\nu+1 / 2}\right)\left(E_{i}^{n+1}+E_{i}^{n}\right) / 2$. Note that the $E$-field used to move the particles is averaged over the macro-steps $(n$ and $n+1)$, which is consistent with the assumption that the electric field evolves in a slower time scale. Using $\bar{j}_{i}^{n+1 / 2}$ in Eq. (8), the energy conservation theorem is recovered as follows:

$$
\begin{aligned}
\sum_{p} \frac{1}{2} m_{p}\left[\left(v_{p}^{n+1}\right)^{2}-\left(v_{p}^{n}\right)^{2}\right] & =\sum_{p} \sum_{\nu} \frac{1}{2} m_{p}\left[\left(v_{p}^{\nu+1}\right)^{2}-\left(v_{p}^{\nu}\right)^{2}\right] \\
& =\sum_{p} \sum_{\nu} \Delta \tau^{\nu} v_{p}^{\nu+1 / 2} q_{p} E\left(x_{p}^{\nu+1 / 2}\right) \\
& =\sum_{p} \sum_{\nu} \Delta \tau^{\nu} v_{p}^{\nu+1 / 2} q_{p} \sum_{i} \frac{E_{i}^{n+1}+E_{i}^{n}}{2} S\left(x_{i}-x_{p}^{\nu+1 / 2}\right) \\
& =\sum_{i} \Delta t \bar{j}_{i}^{n+1 / 2} \frac{E_{i}^{n+1}+E_{i}^{n}}{2} \Delta x \\
& =\sum_{i} \frac{\Delta x}{2} \epsilon_{0}\left[\left(E_{i}^{n+1}\right)^{2}-\left(E_{i}^{n}\right)^{2}\right] .
\end{aligned}
$$

\subsection{Charge conservation}

Standard methods of current and charge assignment in VA do not satisfy the continuity equation (4) [1. Accordingly, charge is not conserved locally, and Gauss's law is violated. Methods for automatic, exact charge conservation for PIC simulations have been developed [26, 31 33]. In general, the charge-conserving schemes are valid only within a given cell, and break when a particle crosses a cell boundary. The problem is usually resolved [26] by splitting current contributions between adjacent cells, as follows:

$$
j_{p}=j_{p 1}+j_{p 2}
$$

where $j_{p}=q_{p} \Delta x_{p} / \Delta t, j_{p 1}=q_{p} \Delta x_{p 1} / \Delta t, j_{p 2}=q_{p} \Delta x_{p 2} / \Delta t, \Delta x_{p 1}$ and $\Delta x_{p 2}$ are segments of the particle displacement within a cell, and $\Delta x_{p 1}+\Delta x_{p 2}=\Delta x_{p}$. By doing so, charge is strictly conserved. However, in our context, this approach would break energy conservation, because it requires the time-centered, orbit-averaged current density shown in the preceding section. Here, we pursue an alternative way of assigning charge-conserving currents, which is to actually make the particle stop at each cell boundary. It can be readily shown (see Appendix Appendix A that this prescription leads to exact charge conservation for each particle sub-step. The property also holds in an orbit-averaged sense. This can be readily seen by taking the orbit-average $\left[\frac{1}{\Delta t} \sum_{\nu=1}^{N_{\nu}} \Delta \tau^{\nu}\right]$ of the continuity equation for each sub-step $(\nu \rightarrow \nu+1)$ :

$$
\frac{\rho_{i+1 / 2}^{\nu+1}-\rho_{i+1 / 2}^{\nu}}{\Delta \tau^{\nu}}+\frac{j_{i+1}^{\nu+1 / 2}-j_{i}^{\nu+1 / 2}}{\Delta x}=0,
$$


and using Eq. 13 and that $\left(\rho_{i+1 / 2}^{n+1}-\rho_{i+1 / 2}^{n}\right)=\sum_{\nu}\left(\rho_{i+1 / 2}^{\nu+1}-\rho_{i+1 / 2}^{\nu}\right)$, to find:

$$
\frac{\rho_{i+1 / 2}^{n+1}-\rho_{i+1 / 2}^{n}}{\Delta t}+\frac{\bar{j}_{i+1}^{n+1 / 2}-\bar{j}_{i}^{n+1 / 2}}{\Delta x}=0 .
$$

This is the continuity equation in terms of the orbit-averaged plasma current. It follows that, once appropriate particle sub-stepping and orbit-averaging schemes are used, our VA implementation simultaneously conserves energy and charge exactly. Unlike the exact energy conservation, charge conservation is ensured regardless of nonlinear convergence tolerance. This is so because the property is built into the particle mover.

\subsection{Particle tunneling and adaptive orbit integration}

The energy- and change-conserving particle mover does not enforce exact momentum conservation. We have found that momentum conservation errors are exacerbated by particle tunneling. This occurs when a particle tunnels through a potential energy barrier, which is statistically unavoidable with a mover employing a fixed time step (see Fig,1). The orbit error caused by particle tunneling affects momentum conservation, because the particle ends up traveling in the wrong direction. While only a few particles experience tunneling, we have found its accuracy impact to be deleterious [This will be demonstrated numerically in Sec.(5)].

Particle tunneling is avoided by our charge-conserving particle mover, as particles are forced to stop at cell boundaries. To further improve the accuracy of our orbit integrator, we have designed an adaptive orbit-integration algorithm. We wish to control the sub-time step $\Delta \tau$ of a particle such that the local truncation error of Eqs.(14) and (15) is below a specified tolerance. This leads to the condition:

$$
\|l e(\Delta \tau)\|_{2}<\varepsilon_{a}+\varepsilon_{r}\left\|r^{0}(\Delta \tau)\right\|_{2}
$$

where $\|\cdot\|_{2}$ denotes the $L_{2}$-norm of enclosed vector, $l e(\Delta \tau)=\frac{(\Delta \tau)^{2}}{2}\left\{a_{p}^{\nu},\left(\frac{\partial a_{p}}{\partial x} v_{p}\right)^{\nu}\right\}$ is a measure of the local truncation error of the discrete orbit equations (see Appendix Appendix B), $\varepsilon_{a}$ and $\varepsilon_{r}$ are absolute and relative tolerances, respectively, and $r^{0}(\Delta \tau) \equiv\left\{v^{0}, a^{0}\right\} \Delta \tau$ is the initial residual. The key to the effectiveness of the approach is in the estimation of $\partial a_{p} / \partial x$ in $l e(\Delta \tau)$. Rather than computing a local estimate based on the current particle position, we compute a cell-based estimate $\partial a_{p} / \partial x \approx \frac{q}{m}\left(E_{i+1}-E_{i}\right) / \Delta x$ (where we have assumed that the particle is located between grid points $i$ and $i+1$ at time level $\nu$ ), and analytically continuate this estimate beyond the cell boundaries. This provides a numerical potential barrier for trapped particles against tunneling, as illustrated in Fig.1. With this estimate, Eq. 19 results in a quadratic equation for an upper limit for $\Delta \tau$. As before, particles stop at cell boundaries to enforce charge conservation.

\subsection{Space filtering}

In the conventional momentum-conserving scheme, finite-grid instabilities result in large energy conservation errors [1]. In energy-conserving schemes, aliasing errors result in the loss of momentum conservation. The aliasing errors arise as the wavelengths of the particle moments, which are sampled in continuous space, can be much shorter than those of moments 


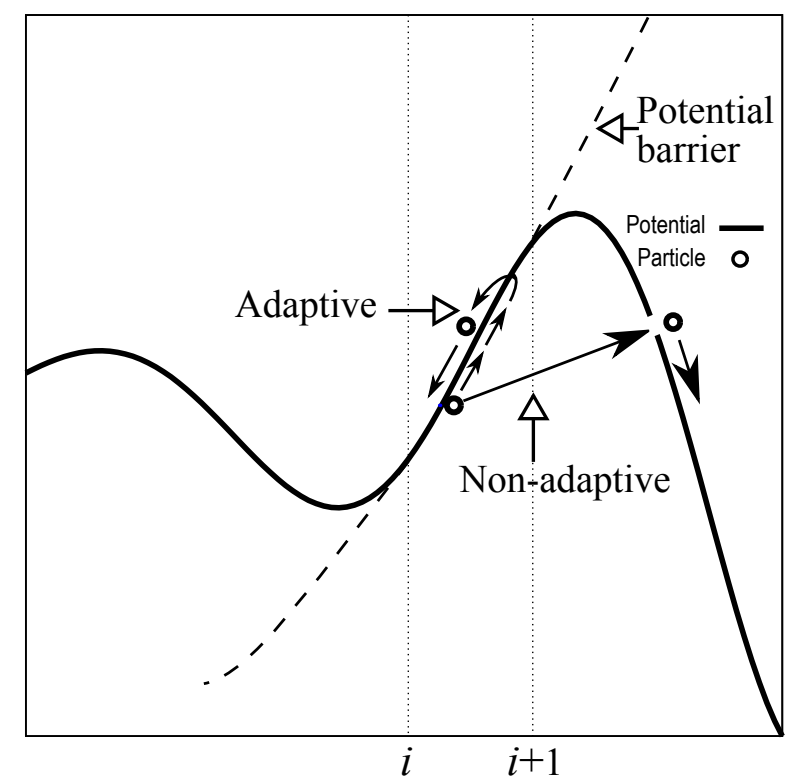

Figure 1: Illustration of particle tunneling through a potential barrier. With charge-conserving, adaptive stepping, a trapped particle is not allowed to tunnel through the potential barrier.

defined on the mesh. Because of the finite number of grid points, mode perturbations with $k \Delta x>\pi$ contribute to the modes with $k \Delta x<\pi$. The particle shape function can be viewed as a low pass filter to reduce aliasing errors: a B-spline of order $m$ has the form of $S_{m}(k)=\left(\sin \frac{1}{2} k \Delta x / \frac{1}{2} k \Delta x\right)^{m}$, which depresses short-wave-length signals. One can achieve further filtering by increasing $m$, but this has disadvantages (for instance, it results in extended stencils which complicate a parallel implementation). Alternatively, one can apply space filtering of grid quantities, which is recommended to further reduce noise signals with $k \Delta x \rightarrow \pi$ [34]. One simple example is the $\cos ^{2}\left(\frac{k \Delta x}{2}\right)$ (binomial) filter in Fourier space. In real space, it is equivalent to replacing a grid quantity $Q_{i}$ with a binomial operator $S M$ such that

$$
S M(Q)_{i}=\frac{Q_{i-1}+2 Q_{i}+Q_{i+1}}{4} .
$$

This is the approach followed in this study. Implementation-wise, two extra operations are introduced in the implicit algorithm. Firstly, the binomial operator is used on the electric field on the mesh, which is then interpolated to the particles. The corresponding particle motion per sub-step is given by

$$
\begin{aligned}
& \frac{x_{p}^{\nu+1}-x_{p}^{\nu}}{\Delta \tau^{\nu}}=v_{p}^{\nu+1 / 2} \\
& \frac{v_{p}^{\nu+1}-v_{p}^{\nu}}{\Delta \tau^{\nu}}=\frac{q_{p}}{m_{p}} S M(E)\left(x_{p}^{\nu+1 / 2}\right)
\end{aligned}
$$


Secondly, the binomial operator is used on the orbit-averaged current density in Ampère's equation:

$$
\epsilon_{0} \frac{E_{i}^{n+1}-E_{i}^{n}}{\Delta t}+S M(\bar{j})_{i}^{n+1 / 2}=\langle\bar{j}\rangle^{n+1 / 2} .
$$

Note that the smoothing does not change the spacial average of $\bar{j}$ on the right-hand-side. It is important to note that both the energy and charge conservation properties still hold with binomial smoothing: one simply needs to realize that, in a periodic domain, $\sum_{i} \bar{j}_{i} S M(E)_{i}=$ $\sum_{i} S M(\bar{j})_{i} E_{i}$, which allows the proof in Eq. 16 and Eq. 117)-(18) to follow through.

\subsection{Equivalence of $V A$ and $V P$}

From the discussion above, it is clear that both Ampere's equation and the charge continuity equation are strictly satisfied. It is therefore easy to deduce that Gauss's law, if satisfied initially, is also strictly satisfied at all times. This follows trivially from the following:

$$
\left.\begin{array}{l}
\epsilon_{0} \frac{E_{i}^{n+1}-E_{i}^{n}}{\Delta t}+\bar{j}_{i}^{n+1 / 2}=\langle\bar{j}\rangle^{n+1 / 2} \\
\frac{\rho_{i+1 / 2}^{n+1}-\rho_{i+1 / 2}^{n}}{\Delta t}+\frac{\bar{j}_{i+1}^{n+1 / 2}-\bar{j}_{i}^{n+1 / 2}}{\Delta x}=0
\end{array}\right\} \Rightarrow\left(\frac{E_{i+1}-E_{i}}{\Delta x}-\rho_{i+1 / 2}\right)^{n+1}=\left(\frac{E_{i+1}-E_{i}}{\Delta x}-\rho_{i+1 / 2}\right)^{n} .
$$

The discrete equivalence of the VA system and VP system is thus evident. Consequently, the implicit energy- and charge-conserving scheme proposed here can also be viewed as an energy-conserving implicit VP formulation, and therefore shares a strong connection to Lewis's explicit energy-conserving scheme[25]. Both methods solve the same system of equations and feature exact charge conservation. Both schemes define $E$ and $\rho$ half grid apart, and the interpolation schemes are identical, namely, the shape function (B-spline) of $E$ is one order lower than that of $\rho$.

There are, however, important differences between the two energy-conserving methods. While Lewis's is explicit and energy-conserving only when $\Delta t \rightarrow 0$, ours is implicit and exactly energy-conserving for arbitrary $\Delta t$. Moreover, unlike explicit PIC schemes (including Lewis's), in which the time step is fixed and usually the same for all particle and field equations, our implicit approach allows different time steps to be used for particles vs. fields. This enables complete flexibility in the orbit integration stage, which allows momentum error control [see Sec.(3.4)].

\section{Formulation of nonlinear residual: nonlinear elimination/particle enslavement}

The goal of this study is to find the nonlinear root of the system of equations (21)-(23). This nonlinearly coupled set of equations can be conceptually formulated as a nonlinear residual of the form:

$$
\mathbf{F}(\mathbf{E}, \boldsymbol{\xi})=0 .
$$

Here, $\mathbf{E}=\left\{E_{i}\right\}$ with $i$ representing mesh points, and $\boldsymbol{\xi}=\left\{x_{p}, v_{p}\right\}$ with $p$ representing particles. Upon convergence, $\mathbf{E}=\left\{E_{i}^{n+1}\right\}$, and $\boldsymbol{\xi}=\left\{x_{p}^{n+1}, v_{p}^{n+1}\right\}$, i.e., the new-time field and particle quantities. In formulating this residual, we have naively taken both particle and field variables as dependent variables. While in principle correct, this formulation has one fundamental limitation, namely, that the residual has inherited the full dimensionality of the kinetic problem. For any iterative solution method, this will result in exceedingly 
large storage requirements. Given that memory is the principal bottleneck in current and future massively parallel computers, pursuing the formulation in Eq.(24) does not appear promising.

There is, however, an alternative, which is to nonlinearly eliminate the kinetic component to the low-dimensional description in terms of fields and moments. The concept of nonlinear elimination is akin to the well-known elimination process in linear equations, and can be explained as follows. Let us consider a nonlinear residual $\mathbf{F}\left(\mathbf{X}_{1}, \mathbf{X}_{2}\right)$, with $\mathbf{X}_{1}, \mathbf{X}_{2}$ two sets of dependent variables. Let us also divide the global residual into two sets of equations, $\mathbf{F}_{1}\left(\mathbf{X}_{1}, \mathbf{X}_{2}\right)=0$ and $\mathbf{F}_{2}\left(\mathbf{X}_{1}, \mathbf{X}_{2}\right)=0$, and assume that $\mathbf{F}_{2}\left(\mathbf{X}_{1}, \mathbf{X}_{2}\right)$ can be straightforwardly written in an explicit form as $\mathbf{X}_{2}=\mathbf{f}_{2}\left(\mathbf{X}_{1}\right)$. It follows that a new residual can be formulated as:

$$
\mathbf{F}_{1}\left(\mathbf{X}_{1}, \mathbf{f}_{2}\left(\mathbf{X}_{1}\right)\right)=\mathbf{G}\left(\mathbf{X}_{1}\right)=0 \text {. }
$$

By construction, the new residual $\mathbf{G}$ has lower dimensionality than the original one $\mathbf{F}$ (and thus requires less storage for the Krylov and Newton iterations), but has the same nonlinear solution. One successful example of the use of nonlinear elimination to significantly reduce the dimensionality of the nonlinear residual in a nontrivial application (the solution of the Fokker-Planck transport equation) is reported in Ref. [35.

In our context, the key point is that the particle equations of motion (21) and (22) can be expressed as $\mathbf{X}_{2}=\mathbf{f}_{2}\left(\mathbf{X}_{1}\right)$ if one considers $\mathbf{X}_{1}=\mathbf{E}$ and $\mathbf{X}_{2}=\boldsymbol{\xi}$. That is, for each particle and for a given $\mathbf{E}$ on the mesh, one can explicitly solve Newton's equations for the newtime particle position and velocity, $\left(x_{p}^{n+1}, v_{p}^{n+1}\right)$. This requires a local nonlinear solve, as the particle position affects the interpolation of the electric field. However the particle equations of motion are not stiff (we employ substepping), and we have found that Picard's method of successive approximations (with a nonlinear tolerance of $10^{-10}$ ) is sufficient to solve the particle equations of motion effectively in each sub-timestep.

As a result, the original residual Eq. (24) can be formally rewritten as a function of $\mathbf{E}$ only:

$$
\mathbf{G}(\mathbf{E})=0 .
$$

This is what we term particle enslavement ( particle quantities do not appear explicitly in the residual). It shows that the full kinetic description can be equivalently formulated in terms of a low-dimensional residual, with solver memory requirements (e.g., nonlinear residuals, Krylov subspace vectors, etc.) comparable to those of a moment/fluid description. (It should be noted that one still needs to save old-time and new-time particle quantities as auxiliary variables to compute the nonlinear residual.) The concept is not restricted to electrostatic $\mathrm{PIC}$, and can be trivially generalized to an electromagnetic formulation with $\mathbf{X}_{1}=\left\{E_{i}, B_{i}\right\}$ and $\mathbf{X}_{2}$ as above. Note that particle enslavement allows complete flexibility in the particle integration step, $\mathbf{X}_{2}=\mathbf{f}_{2}\left(\mathbf{X}_{1}\right)$. Since this operation is segregated in the calculation of the residual $\mathbf{G}$, it is ideally suited to exploit heterogeneous computing architectures such as general-purpose graphics processing units (GPGPUs). This will be explored in future work. Finally, we note that this flexibility has enabled us to explore the advanced features of our orbit integrator, namely, particle sub-stepping, orbit averaging, temporal adaptivity, and charge conservation.

Finding the root of the enslaved residual Eq. 26. is not a simple task, because nonlinear couplings are nonlocal on the mesh, and are very nontrivial due to the complex orbit 
integration/averaging step. Jacobian-free Newton-Krylov (JFNK) methods present important advantages for this application, which we discuss briefly below. The motivated reader can find extensive discussions on this approach elsewhere [22]. Newton's method solves the nonlinear system $\mathbf{G}(\mathbf{x})=\mathbf{0}$ (with $\mathbf{x}=\mathbf{E}$ ) iteratively by inverting linear systems of the form:

$$
\left.\frac{\partial \mathbf{G}}{\partial \mathbf{x}}\right|^{(k)} \delta \mathbf{x}^{(k)}=-\mathbf{G}\left(\mathbf{x}^{(k)}\right),
$$

with $\mathbf{x}^{(k+1)}=\mathbf{x}^{(k)}+\delta \mathbf{x}^{(k)}$, where $(k)$ denotes the nonlinear iteration number. Nonlinear convergence is achieved when:

$$
\left\|\mathbf{G}\left(\mathbf{x}^{(k)}\right)\right\|_{2}<\epsilon_{a}+\epsilon_{r}\left\|\mathbf{G}\left(\mathbf{x}^{(0)}\right)\right\|_{2}=\epsilon_{t},
$$

where $\|\cdot\|_{2}$ is the $L_{2}$-norm, $\epsilon_{a}=\sqrt{N} \times 10^{-15}$ (with $N$ the total number of degrees of freedom) is an absolute tolerance to avoid converging below round-off, $\epsilon_{r}$ is the Newton relative convergence tolerance (set to $10^{-8}$ in this work, unless otherwise specified), and $\mathbf{G}\left(\mathbf{x}^{(0)}\right.$ ) is the initial residual.

Such linear systems are solved iteratively with Krylov methods, which only require matrix-vector products to proceed. Because the linear system matrix is a Jacobian matrix, such matrix-vector products can be implemented Jacobian-free using the Gateaux derivative:

$$
\left.\frac{\partial \mathbf{G}}{\partial \mathbf{x}}\right|^{(k)} \mathbf{v}=\lim _{\epsilon \rightarrow 0} \frac{\mathbf{G}\left(\mathbf{x}^{(k)}+\epsilon \mathbf{v}\right)-\mathbf{G}\left(\mathbf{x}^{(k)}\right)}{\epsilon},
$$

where in practice a small but finite $\epsilon$ is employed (p. 79 in [22]). Thus, the evaluation of the Jacobian-vector product only requires the function evaluation $\mathbf{G}\left(\mathbf{x}^{(k)}+\epsilon \mathbf{v}\right)$, and there is no need to form or store the Jacobian matrix. This, in turn, allows for a memory-efficient implementation.

An inexact Newton method [36] is used to adjust the convergence tolerance of the Krylov method at every Newton iteration according to the size of the current Newton residual, as follows:

$$
\left\|J^{(k)} \delta \mathbf{x}^{(k)}+\mathbf{G}\left(\mathbf{x}^{(k)}\right)\right\|_{2}<\zeta^{(k)}\left\|\mathbf{G}\left(\mathbf{x}^{(k)}\right)\right\|_{2}
$$

where $\zeta^{(k)}$ is the inexact Newton parameter and $J^{(k)}=\left.\frac{\partial \mathbf{G}}{\partial \mathbf{x}}\right|^{(k)}$ is the Jacobian matrix. Thus, the convergence tolerance of the Krylov method is loose when the Newton state vector $\mathbf{x}^{(k)}$ is far from the nonlinear solution, and tightens as $\mathbf{x}^{(k)}$ approaches the solution. Superlinear convergence rates of the inexact Newton method are possible if the sequence of $\zeta^{(k)}$ is chosen properly (p. 105 in [22]). Here, we employ the prescription:

$$
\begin{aligned}
\zeta^{A(k)} & =\gamma\left(\frac{\left\|\mathbf{G}\left(\mathbf{x}^{(k)}\right)\right\|_{2}}{\left\|\mathbf{G}\left(\mathbf{x}^{(k-1)}\right)\right\|_{2}}\right)^{\alpha}, \\
\zeta^{B(k)} & =\min \left[\zeta_{\max }, \max \left(\zeta^{A(k)}, \gamma \zeta^{\alpha(k-1)}\right)\right], \\
\zeta^{(k)} & =\min \left[\zeta_{\max }, \max \left(\zeta^{B(k)}, \gamma \frac{\epsilon_{t}}{\left\|\mathbf{G}\left(\mathbf{x}^{(k)}\right)\right\|_{2}}\right)\right],
\end{aligned}
$$

with $\alpha=1.5, \gamma=0.9$, and $\zeta_{\max }=0.8$. The convergence tolerance $\epsilon_{t}$ is defined in Eq.(27). In this prescription, the first step ensures superlinear convergence (for $\alpha>1$ ), the second avoids volatile decreases in $\zeta_{k}$, and the last avoids oversolving in the last Newton iteration. 
A further advantage of Krylov methods is that they can be preconditioned by considering the alternate (but equivalent) systems $J^{(k)}\left(P^{(k)}\right)^{-1} P^{(k)} \delta \mathbf{x}^{(k)}=-\mathbf{G}^{(k)}$ (right preconditioning) or $\left(P^{(k)}\right)^{-1} J^{(k)} \delta \mathbf{x}^{(k)}=-\left(P^{(k)}\right)^{-1} \mathbf{G}^{(k)}$ (left preconditioning). Such preconditioned systems can be straightforwardly and efficiently implemented in the Krylov algorithm as two consecutive matrix-vector products. A crucial feature of preconditioning is that, while it can substantially improve the convergence properties of the Krylov iteration if $\left(P^{(k)}\right)^{-1} \approx\left(J^{(k)}\right)^{-1}$, it does not alter the solution of the Jacobian system upon convergence (because the solution $\delta \mathbf{x}^{(k)}$ of the preconditioned system is the same as that of the original system). Therefore, one can explore suitable approximations in the preconditioner for efficiency purposes without compromising the accuracy of the converged result.

For this study, we have employed unpreconditioned JFNK (with $P^{(k)}$ the identity operator) to demonstrate the feasibility and the accuracy properties of our fully implicit electrostatic PIC implementation. Despite the lack of a preconditioner, we show in the next section that large CPU speedups over explicit approaches are still possible in 1D, demonstrating the potential of the approach. Multidimensional applications will require effective preconditioning, which will be the subject of future work.

\section{Numerical examples}

To demonstrate the accuracy and performance of the present algorithm, we use three standard electrostatic test cases: Langmuir wave, two-stream instability, and ion acoustic wave. We first show that a simple CN mover (i.e., without sub-stepping) can accurately simulate the behavior of Langmuir waves, including cold plasma oscillations and Landau damping in a warm plasma. We then simulate a two-stream instability, demonstrating the effects of charge conservation and adaptive sub-stepping on momentum conservation. In the IAW case, in which large implicit time steps $\left(\omega_{p e} \Delta t \gg 1\right)$ can be taken, we discuss the impact of the particle mover on the accuracy of long-time integration, and demonstrate that moderate CPU gains of the implicit scheme vs. the explicit scheme in the $k \lambda_{D} \ll 1$ limit are possible.

In all the cases, we assume a homogeneous collisionless electrostatic plasma equilibrium with some initial perturbation

$$
f_{\alpha}(x, v, t=0)=f_{\alpha 0}(v)\left[1+a \cos \left(\frac{2 \pi n_{h}}{L} x\right)\right]
$$

where $f_{\alpha 0}$ is the initial velocity distribution of species $\alpha, a$ is the perturbation level, $L$ is the domain size, and $n_{h}$ is the mode number of the perturbation $\left(n_{h}=1\right.$ by default). The computational domain is one-dimensional, featuring a uniform grid and periodic boundary conditions. All the temporal and spatial quantities are normalized by the inverse plasma frequency $\left(1 / \omega_{p e}\right)$ and the Debye length $\left(\lambda_{D}\right)$, respectively. Other parameters are provided in specific test cases below. To verify the simulations using the implicit algorithm, we use either analytical solutions, e.g., linear damping or instability rates, or results from the conventional explicit momentum-conserving VP PIC simulations. By default, binomial spatial smoothing [Eq. 201] is used; first-order B-spline is used for interpolating current density $j$ in the implicit scheme, and second-order B-spline is used for interpolating charge density $\rho$ in the explicit scheme. 
implicit $-\quad$ explicit

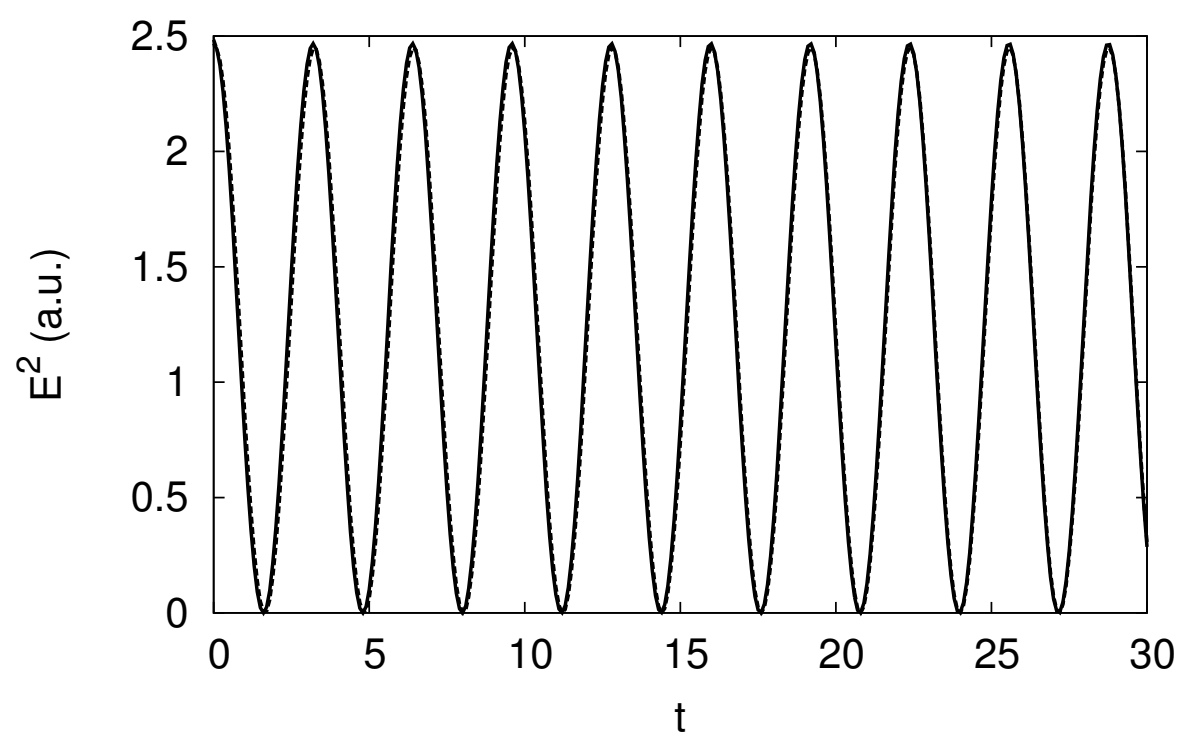

Figure 2: Langmuir wave in a cold plasma. Leapfrog and simple CN are used in explicit and implicit schemes respectively. Both schemes use a small time step $(\Delta t=0.1)$. The two curves depict the total electric field energy $E^{2}=\sum_{i} E_{i}^{2}$ and are very close to each other. The theoretical plasma frequency $\left(\omega_{p e}=1\right)$ is accurately reproduced.

\subsection{Langmuir wave}

We first consider a cold plasma Langmuir wave, in which electrons are perturbed and oscillate collectively around stationary and uniform ions. Simulations are performed by both explicit and implicit schemes using the same set of simulation parameters: $L=2 \pi$, $N_{x}=32, N_{p}=2000, \Delta t=0.1, a=0.01$, which are the domain size, number of grid points, number of particles, the time step, and the perturbation level [see Eq.[30]], respectively. Figure (22), shows that the leapfrog and $\mathrm{CN}$ schemes agree closely with each other. In these simulations, we resolve well the plasma period, which is the only timescale of the problem.

Next, we consider electron Landau damping of Langmuir waves in a warm plasma, which is a pure kinetic effect. Given Maxwellian electrons and no contribution from ions, the wave dispersion relation from linear theory is [37]:

$$
1+\frac{1}{k^{2}}\left[1+\frac{\omega}{\sqrt{2} k} Z\left(\frac{\omega}{\sqrt{2} k}\right)\right]=0,
$$

where $Z$ is the dispersion function of Fried and Conte. Solving Eq.(31) for a given real $k$ provides a complex solution $(\omega+i \gamma)$, in which $\omega$ is the wave frequency and $\gamma$ is the wave damping rate.

We initialize the problem with a perturbation of a Maxwellian distribution $f_{e 0}$. The simulation parameters are $L=4 \pi, N_{x}=32, N_{p}=40000, a=0.05$, defined as before. Using $k=0.5$ in Eq.(31), we find $\omega=1.415$ and $\gamma=0.154$. Note that Landau damping, which mostly affects resonant particles with $v_{p} \sim \omega / k$, occurs in a longer timescale than 

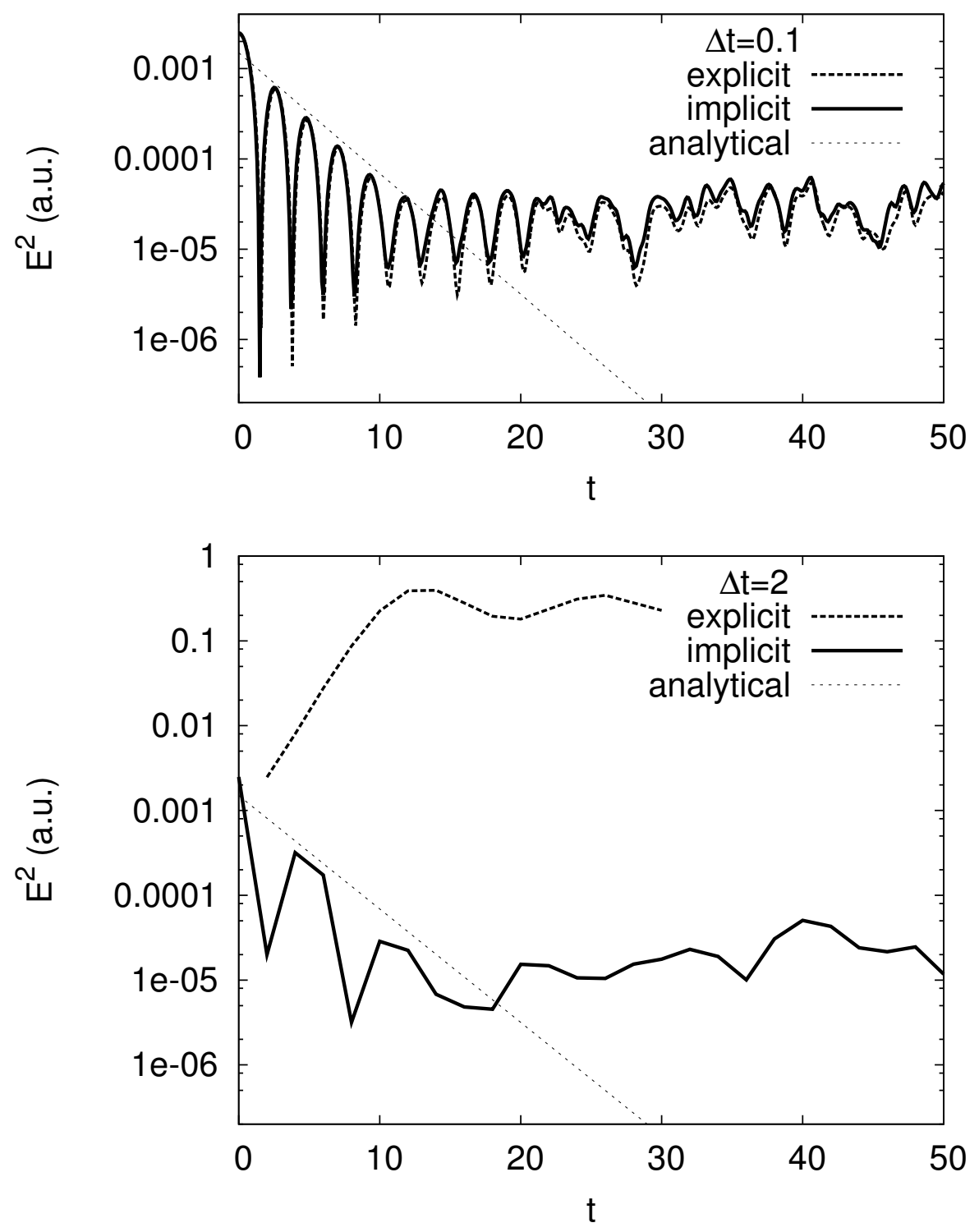

Figure 3: Landau damping test case. Top: Good agreement between the explicit and the implicit schemes is seen for $\Delta t=0.1$. Bottom: Accurate damping rate is produced by the implicit scheme for $\Delta t=2$ (i.e., $\omega_{p} \Delta t=2$ and $\gamma \Delta t \simeq 0.3$ ) even though the plasma frequency is underresolved. For this time step, the explicit scheme is CFL unstable. 


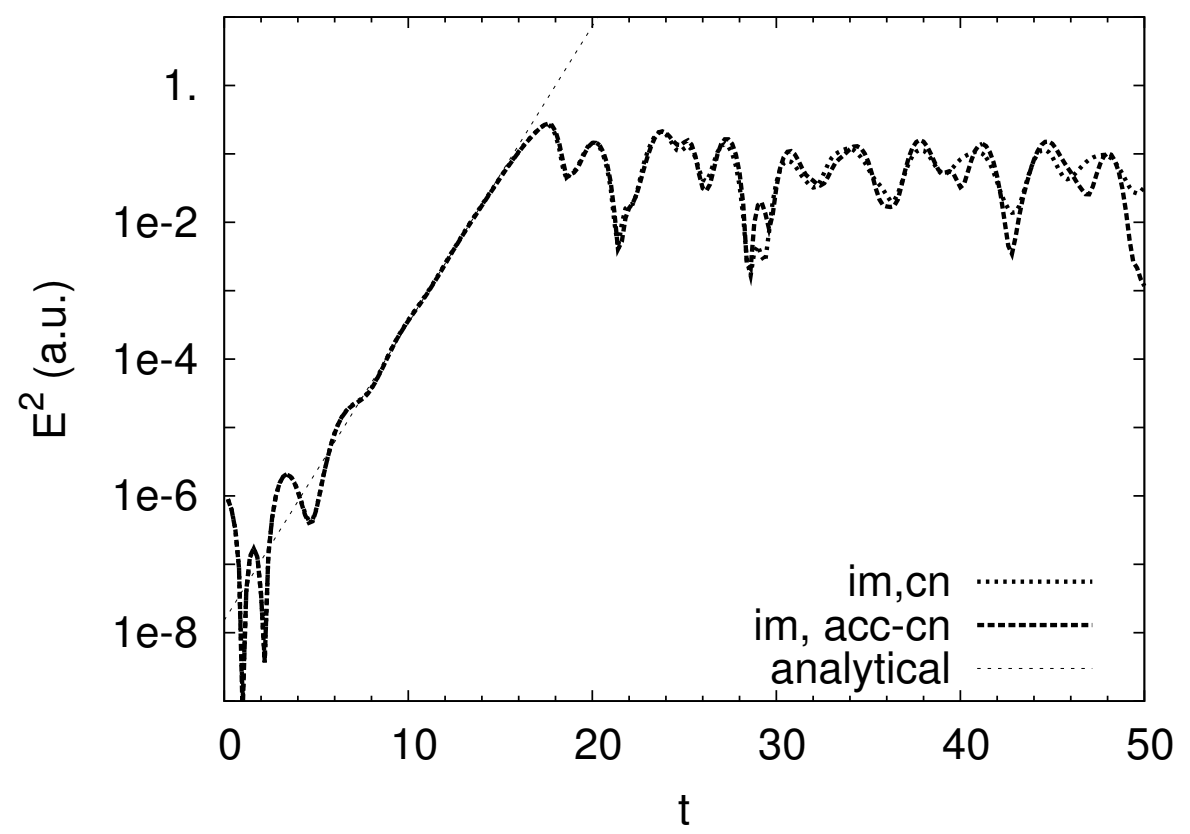

Figure 4: Semi-log scale graph of the electric field energy for the two-stream instability, obtained with the simple CN mover and the ACC CN mover. Both simulations use $\Delta t=0.2$. Numerical growth rates are seen to agree very well with the analytical one $(\gamma=0.5)$.

plasma oscillations, so that there is a separation of timescales in this problem. We first consider a small time step $\Delta t=0.1$, which resolves the plasma wave period. We performed both explicit and implicit simulations under identical conditions. Figure (3) a compares the results with the analytical growth rate. We see good agreement between the two methods, and the numerical damping rate agrees well with the theoretical one. When we increase the time step to $\Delta t=2$ [Fig. [3] b] (such that the plasma period is under-resolved, but the damping rate is still well resolved), the explicit scheme is unstable due to the violation of the CFL condition. The implicit scheme, on the other hand, is stable, and accurately reproduces the damping rate. We note that only the simple CN mover is employed in these implicit simulations, which enforce no local charge conservation. The importance of local charge conservation will be demonstrated for longer term simulations in the following sections.

\subsection{Two-stream instability}

We consider two equal counter-streaming electron beams in a stationary and uniform ion background. The analytical dispersion relation is [37]

$$
\frac{1}{\left(\omega-k v_{b}\right)^{2}}+\frac{1}{\left(\omega+k v_{b}\right)^{2}}=1
$$

where $v_{b}$ is the beam speed. The simulation is performed for $L=2 \pi, v_{b}=\sqrt{3} / 2, N_{x}=64$, $N_{p}=10000, \Delta t=0.2$, and $a=0.005$, as defined previously. Figure (4) depicts the evolution of the electrostatic energy, showing that the numerical growth rate agrees very well with the analytical one with both the simple $\mathrm{CN}$ mover and the adaptive-charge-conserving (ACC) CN mover. 

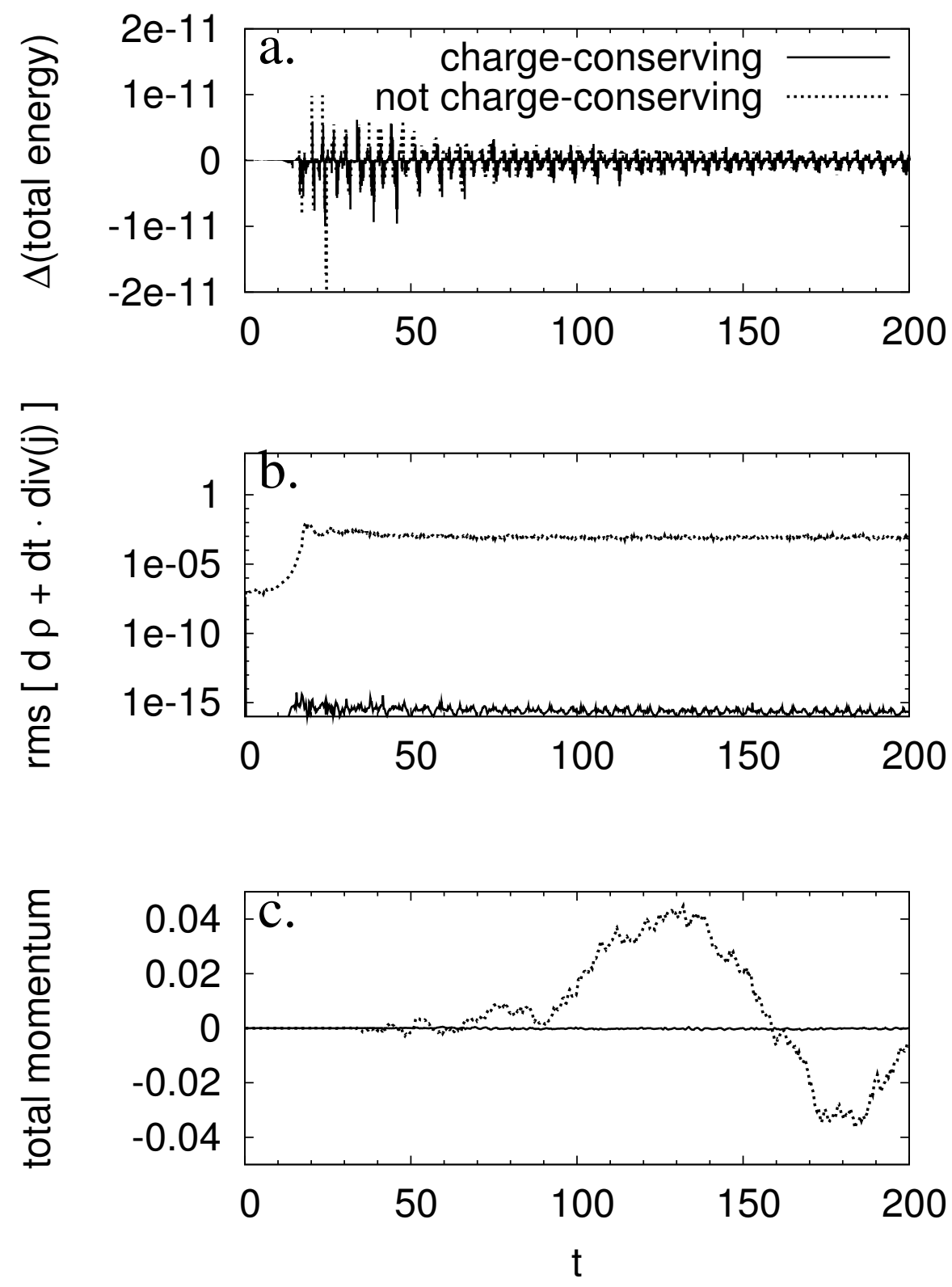

Figure 5: Comparison of the ACC CN and the simple CN particle movers. Time histories of the change in total energy, the root mean square of the charge continuity equation, and the total momentum are shown in panels a,b, and c, respectively. 
This test case is ideally suited for exploring the properties of the ACC CN mover vs. simple CN. For this, the conserved quantities of the system (energy, charge, and momentum) are monitored using both $\mathrm{CN}$ movers. In Fig.(5)a, we see that both $\mathrm{CN}$ movers conserve the total energy commensurately with the nonlinear tolerance employed $\left(\epsilon_{t}=10^{-8}\right)$, as expected. Figure (5) b depicts the root mean square of the discrete charge conservation equation at every cell, and confirms that the ACC mover conserves charge exactly, while the simple CN mover results in significant charge conservation errors. Finally, Fig. (5)c demonstrates the substantial improvement in the conservation of total momentum achieved by the ACC mover vs. the simple $\mathrm{CN}$ mover, particularly in the nonlinear regime.

\subsection{Ion acoustic wave}

Simulating the ion acoustic wave requires considering both electron and ion dynamics. The wave is excited by an ion density perturbation, and is propagated by the compression and expansion caused by the thermal motion of ions, as well as by incomplete Debye shielding due to the thermal motion of electrons. Adding the ion contribution to the dielectric constant [cf. Eq.311], the dispersion relation of ion acoustic wave can be written as [37]

$$
1+\frac{1}{k^{2}}\left[1+\frac{\omega}{\sqrt{2} k} Z\left(\frac{\omega}{\sqrt{2} k}\right)\right]+\frac{\sqrt{r_{T}}}{k^{2}}\left[1+\frac{\sqrt{r_{T} r_{m}} \omega}{\sqrt{2} k} Z\left(\frac{\sqrt{r_{T} r_{m}} \omega}{\sqrt{2} k}\right)\right]=0,
$$

where $r_{T}=T_{e} / T_{i}$ is the ratio of electron and ion temperatures, and $r_{m}=m_{i} / m_{e}$ is the ratio of ion and electron masses. In regimes with $r_{T} \gg 1$ and $r_{m} \gg 1$, the IAW frequency is much smaller than the plasma frequency $\left(\omega \ll \omega_{p e}\right)$, and the wavelength is much longer than the Debye length $\left(k \lambda_{D} \ll 1\right)$, which make the IAW a truly multiscale problem.

The main goals of this section are to examine the long-term accuracy of the implicit algorithm employing different particle movers, and to provide an accuracy and efficiency comparison with a standard explicit VP algorithm with a leap-frog mover. We perform implicit and explicit simulations under identical conditions with $r_{T}=10^{4}, r_{m}=200, a=0.2$, $L=10, N_{x}=32, N_{p}=128000$. Note that electrons are much faster than ions for these parameters. Therefore, both stability and accuracy will be largely controlled by electron dynamics.

We first consider four different implicit movers: a single-step CN mover with $\Delta t=\Delta \tau=$ 0.25 and $\Delta t=\Delta \tau=1$, a sub-stepping CN mover with $\Delta t=4 \Delta \tau=1$ (i.e., 4 particle substeps), and an adaptive charge-conserving CN mover. Simulation results with these movers for IAW are depicted in Fig.(6). Figure (6) a depicts the evolution of the ion kinetic energy, and motivates several important observations. Firstly, the single-step CN with $\Delta t=1$ fails to capture the long-term IAW behavior. This is because the average distance that an electron travels is larger than a cell size, namely $k_{\max } v_{t h e} \Delta t>1$. In this regime, Debye shielding is not captured accurately, as predicted by theory [30]. Decreasing the time step to ensure $k_{\text {max }} v_{\text {the }} \Delta t<1$ improves the simulation results (CN with $\Delta t=0.25$ ), as expected, albeit still with significant errors. Particle sub-stepping such that $k_{\text {max }} v_{\text {the }} \Delta \tau<1$ also improves the simulation results, as is demonstrated by the sub-stepping $\mathrm{CN}$ run, but it is still not satisfactory. The implicit solve with ACC mover is the only one that is able to accurately capture the dynamics despite the long $\Delta t$ employed, as will be shown later in this section.

Figure (6) b shows the evolution of the electron kinetic energy. The relative magnitude of the perturbation in the electron kinetic energy is much smaller than that in the ion kinetic 

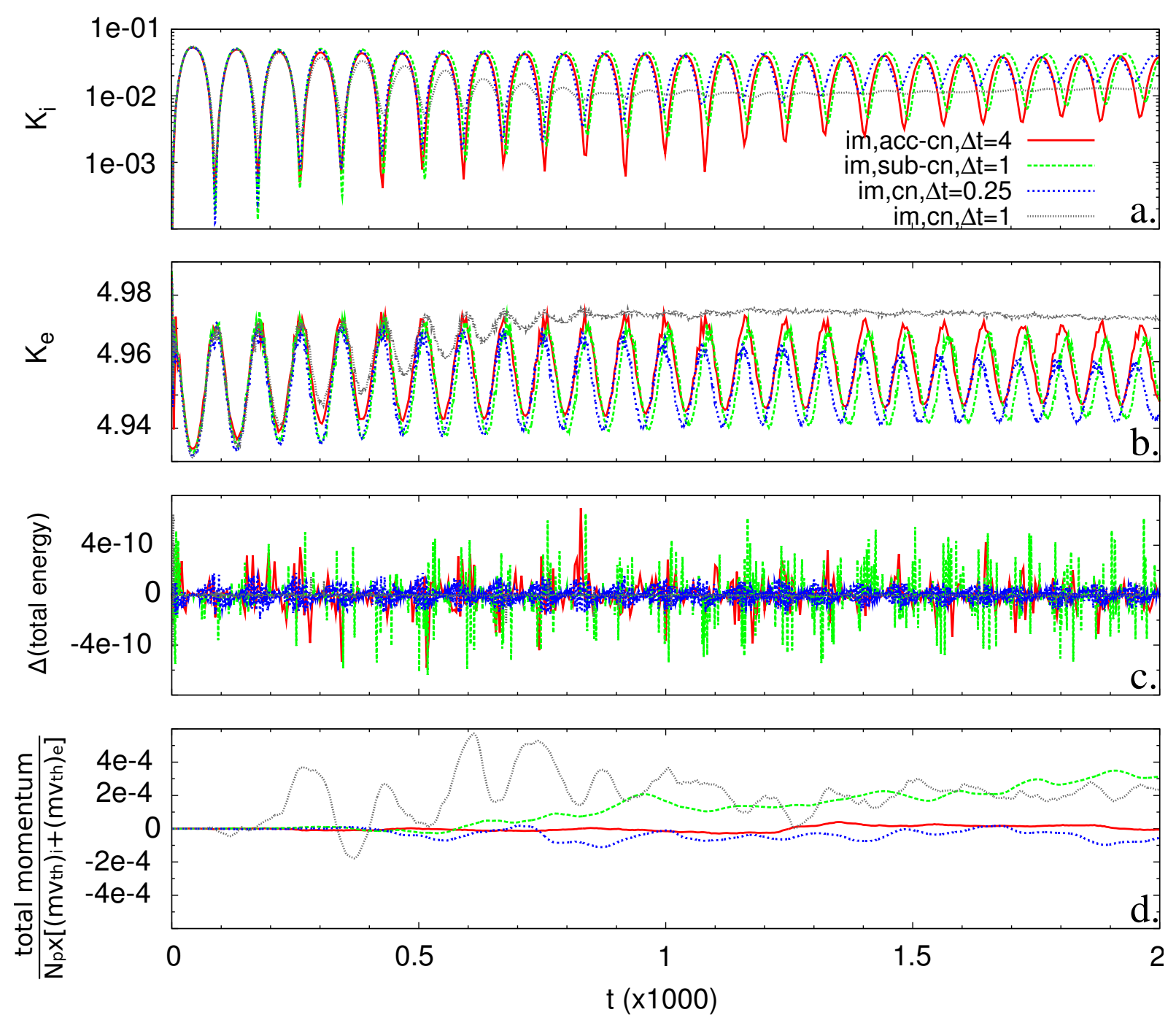

Figure 6: Accuracy test of the implicit algorithm using three movers: simple CN mover (cn), sub-stepped CN mover (sub-cn), and adaptive-charge-conserving CN mover (acc-cn). a) Time history of ion kinetic energy shows the impact of using fixed-time step movers in a long-time simulation. b) Time history of electron kinetic energy shows the importance of using small (sub-)time steps to capture the electron dynamics, which exhibits relatively small variations. c) Total energy is well conserved in all implicit simulations. d) Momentum conservation is substantially improved when particle orbits are accurately calculated using the charge-conserving self-adaptive CN mover. 


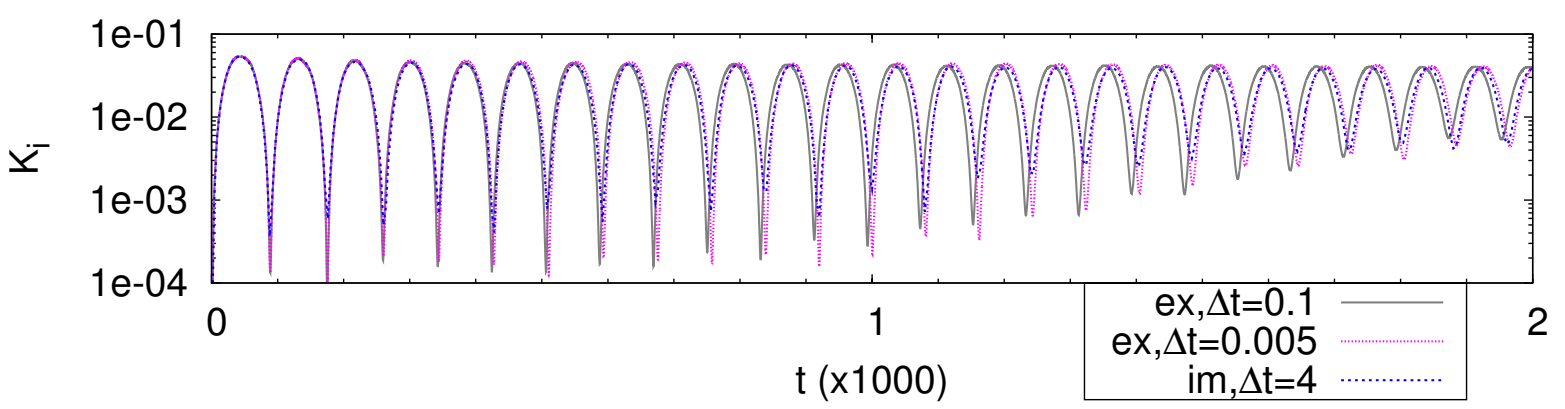

Figure 7: Ion kinetic energy $\left[K_{i}=\sum_{p}\left(\frac{1}{2} m v_{p}^{2}\right)_{i}\right]$ obtained by implicit (with ACC-CN mover) and explicit simulations.

energy, suggesting that small errors in the electron kinetics are sufficient to ruin the accuracy of the ion wave.

Figure (6) $\mathrm{c}$ and (6) d show the time evolution of the conserved quantities of the system, namely, total energy and total momentum, respectively. Clearly, energy is conserved exactly, while momentum is not. Similar to the two-stream instability case [Fig.(50], we see significant improvement in the momentum conservation when exact charge conservation is enforced by the ACC mover. With other movers, momentum errors accumulate in time, and the system can develop nonphysical drifts, which eventually compromise the accuracy of the IAW dynamics.

It is instructive at this point to compare the implicit ACC-CN solver with the explicit VP solver. For the latter, we have employed explicit time steps of $\Delta t=0.1$ and $\Delta t=0.005$, both of which resolve well the plasma frequency (i.e., the CFL condition is satisfied). The results of the comparison are shown in Fig.(7). Most strikingly, only the explicit simulation with the smaller time step agrees well with the implicit ACC solver, despite the fact that the latter takes a very large time step for the field equations $(\Delta t=4$, or $\omega \Delta t \simeq 0.14$, 800 times larger than the explicit one). This suggests that $v_{t h}$-based CFL limits, while sufficient for stability, may not be enough to guarantee accuracy. In fact, a $v_{t h}$-based CFL can introduce large orbit integration errors for fast electrons, which may accumulate in time. The only way to control these long-term errors is to further reduce the explicit time step to resolve the fast electron orbits, thus exacerbating the computational efficiency issues of explicit PIC.

The robustness of the implicit solver with respect to the nonlinear tolerance $\varepsilon_{t}$ for the various movers is examined next. This is of interest because the level of energy conservation depends on $\varepsilon_{t}$. Figure (8) shows the comparison of simulations using two nonlinear tolerances: $\epsilon_{t}=10^{-8}$ and $\epsilon_{t}=10^{-6}$. Even though the energy conservation is not exact with $\varepsilon_{t}=10^{-6}$, the total energy is still conserved well (with errors per time step $\sim 10^{-7}$ ). We find that the ACC mover is the most robust with respect to changes in the nonlinear tolerance. This again stresses the point that orbit accuracy, charge-conservation, and the avoidance of particle tunneling are key to the overall long-term accuracy of the implicit solver.

\subsection{CPU gain of the implicit scheme vs. the explicit scheme}

Having demonstrated the accuracy of the present implicit scheme, we proceed to argue and demonstrate by numerical experiment that the implicit scheme can also be significantly more efficient than the explicit one, even without a preconditioner. 


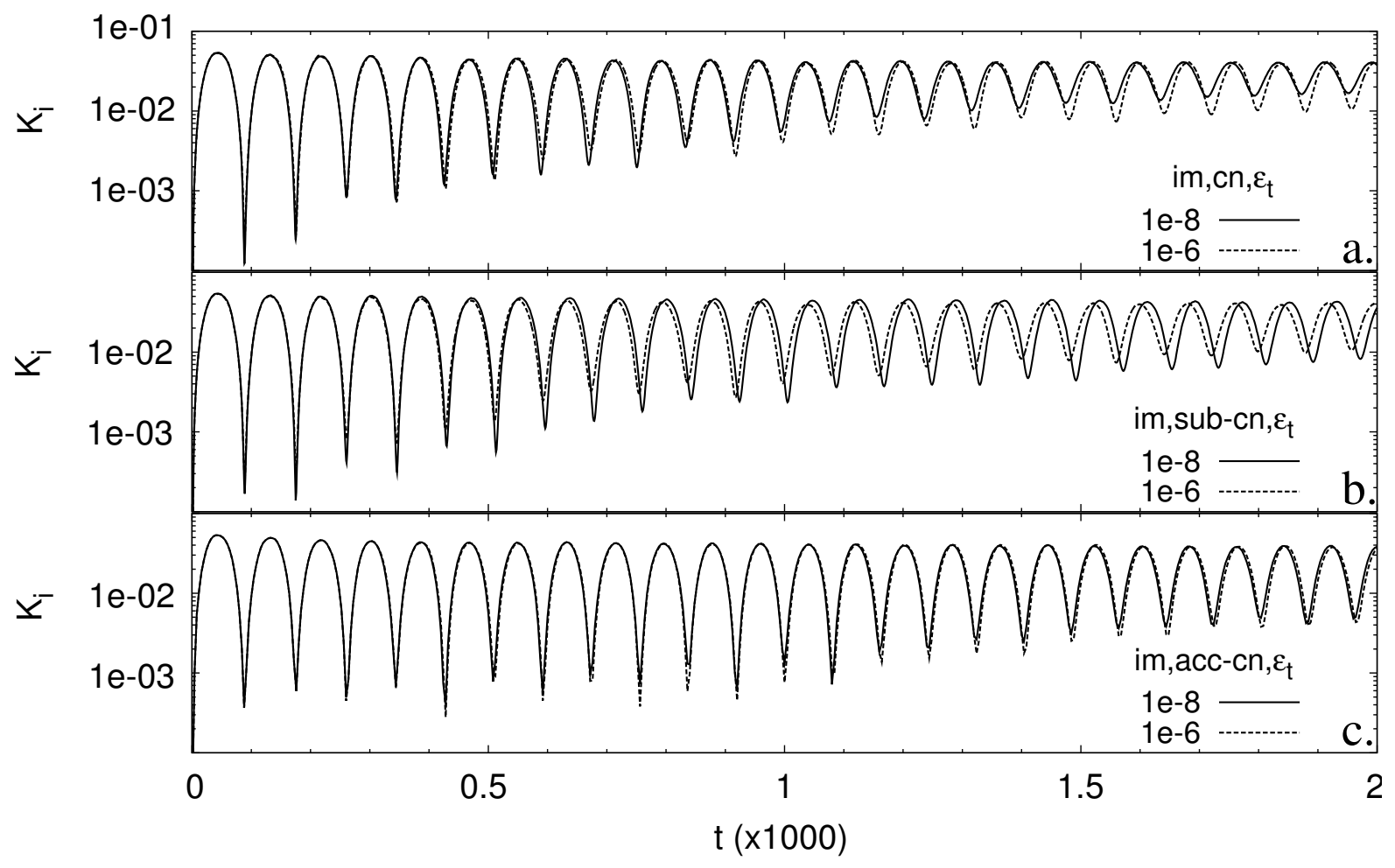

Figure 8: Comparison of the ion kinetic energy history using two nonlinear tolerances $\left(10^{-8}\right.$ and $\left.10^{-6}\right)$ in the implicit simulations. Three particle movers are tested: simple CN mover ( $\mathrm{cn}$ ), sub-stepping CN mover (sub$\mathrm{CN}$ ) and adaptive-charge-conserving $\mathrm{CN}$ mover (ACC-CN). The cn mover and sub-cn mover show strong sensitivity to the nonlinear tolerance (a, b sub-panels), illustrating the effect of accumulated errors in particle orbits. In contrast, the acc-cn particle mover is mostly insensitive to changes in the nonlinear tolerance. 
As has been demonstrated, the proposed energy- and charge-conserving implicit PIC scheme is not limited to the stringent stability constrains featured by conventional explicit PIC schemes, namely, $\omega_{p e} \Delta t<1$ and $\Delta x<\lambda_{D}$. Instead, we can choose the time step to resolve the dynamical timescale of interest, which is in many cases much slower than the plasma frequency. Similarly, we can choose the grid size to resolve the relevant spatial scales, which can be much larger than the Debye length. Thus, there is significant potential for CPU speed-up of implicit vs. explicit PIC schemes.

We have designed the particle integration scheme to follow orbits accurately. At first glance, it may appear that the cost of orbit sub-stepping would offset any potential efficiency gains of the implicit approach. This would certainly be the case if electron sub-time steps were comparable to their explicit CFL. However, sub-time steps in our implicit scheme are not limited by the CFL condition. Instead, sub-steps are typically $\Delta \tau \lesssim \Delta x / v_{p}$, which can still be much larger than the explicit CFL when $\Delta x \gg \lambda_{D}$, and only become restrictive for very few fast particles. It follows that the implicit scheme should still able to deliver large CPU gains with respect to explicit, despite particle sub-stepping.

A back-of-the-envelope estimate confirms that this is the case when $k \lambda_{D} \ll 1$ (the relevant physics regime in many applications of interest). We begin to compute the expected CPU gain by estimating the CPU cost for a given PIC solver as:

$$
C P U=\frac{\Delta T}{\Delta t} n_{p}\left(\frac{L}{\Delta x}\right)^{d} C,
$$

where $\Delta T$ is the time-span of the simulation, $n_{p}$ is the number of particles per cell, $(L / \Delta x)$ is the number of cells, $d$ is the number of physical dimensions, and $C$ is the computational complexity of the solver employed, measured in units of a standard explicit PIC VP leapfrogged time step. The implicit-to-explicit speedup is thus given by:

$$
\frac{C P U_{e x}}{C P U_{i m}} \sim\left(\frac{\Delta x_{i m}}{\Delta x_{e x}}\right)^{d}\left(\frac{\Delta t_{i m}}{\Delta t_{e x}}\right) \frac{1}{C_{i m}} .
$$

For simplicity, we assume that all particles take a fixed sub-timestep $\Delta \tau$ in the implicit scheme, and that the cost of one time step with the explicit PIC solver is comparable to that of a single implicit sub-step (a good approximation when both are dominated by the cost of moving particles). It follows that $C_{i m} \sim N_{F E}(\Delta t / \Delta \tau)_{i m}$, i.e., the cost of the implicit solver exceeds that of the explicit solver by the number of function evaluations per time step $N_{F E}$ multiplied by the number of particle sub-steps $(\Delta t / \Delta \tau)_{i m}$. The number of function evaluations per time step $N_{F E}$ is roughly equal to the sum of the number of Krylov iterations plus the number of Newton iterations in a given time step. If an optimal preconditioner is available, $N_{F E}$ can be made independent of problem dimension and size (see e.g. [38 40] for examples in the context of fluid modeling of plasmas).

Assuming typical values for $\Delta \tau_{i m} \sim 0.1 \Delta x / v_{t h}, \Delta t_{e x} \sim 0.1 / \omega_{p e}, \Delta x_{i m} \sim 0.2 / k$, and $\Delta x_{e x} \sim \lambda_{D}$, we find that the CPU speedup scales as:

$$
\frac{C P U_{e x}}{C P U_{i m}} \sim \frac{1}{\left(k \lambda_{D}\right)^{d+1}} \frac{1}{N_{F E}}
$$


Table 1: Efficiency study of explicit vs. implicit PIC using the IAW example. We have fixed the number of particles per cell to 4000, and the resolution of the implicit runs to 32 mesh points.

\begin{tabular}{cccccc}
$L$ & $k \lambda_{D}$ & $\frac{N_{x}^{e x}}{N_{x}^{i m}}$ & $\frac{\Delta t_{i m}}{\Delta t_{e x}}$ & $N_{F E}$ & $\frac{C P U_{e x}}{C P U_{i m}}$ \\
\hline \hline 10 & 0.628 & 1 & 50 & 13.7 & 0.25 \\
20 & 0.314 & 2 & 100 & 20 & 0.58 \\
40 & 0.157 & 4 & 200 & 31.2 & 0.95 \\
80 & 0.078 & 8 & 200 & 35.8 & 2.18 \\
160 & 0.039 & 16 & 200 & 43.6 & 5.41 \\
160 & 0.039 & 16 & 400 & 72.1 & 3.64 \\
320 & 0.02 & 32 & 200 & 49.6 & 15.4 \\
320 & 0.02 & 32 & 400 & 67.6 & 11.96
\end{tabular}

which predicts that large gains are possible when $k \lambda_{D} \ll 1$, particularly in multidimensional applications, but only if $N_{F E}$ is bounded. The latter point underscores the importance of developing suitable preconditioning strategies. This will be the subject of future work.

We proceed to verify the scaling of Eq. (35) with the IAW example. We employ various values of $k \lambda_{D}$ with both implicit and explicit PIC. Performance results are summarized in Table 1. In all these runs, we have fixed the number of cells in the implicit runs to 32 , and we have used a constant number of particles per cell (4000). Explicit time steps and resolutions are chosen for stability, not accuracy. Since the latter are much more restrictive (as shown earlier in this study), the results in Table 11 should be considered as a conservative lower bound in the efficiency gain potential. As we decrease $k \lambda_{D}$ (i.e., we increase the domain size), we confirm that the implicit-to-explicit CPU ratio $C P U_{e x} / C P U_{i m}$ (last column of Table 1) increases linearly with the domain size. This is consistent with the prediction of Eq. (35) when one realizes that the number of function evaluation $N_{F E}$ also increases linearly with the domain size. This results from our unpreconditioned JFNK implementation combined with the fact that we have also increased the implicit time step as the domain size grows. Nevertheless, Table 1 shows that moderate CPU gains (up to a factor of 15 for $k \lambda_{D}=0.02$ ) can be achieved even without a preconditioner, exemplifying the potential of the implicit PIC approach for efficient, kinetic plasma simulation.

\section{Conclusions}

In this study, we have undertaken the task of implementing and demonstrating the accuracy and algorithmic properties of a fully implicit particle-in-cell solver for plasma simulation. We have focused on a 1D Vlasov-Ampère electrostatic model as a proof of principle, for which we have developed an exactly energy-conserving, orbit-averaged discrete formulation. Exact charge conservation is achieved by an adaptive, charge-conserving, sub-stepping particle mover.

The nonlinear residual is converged using an unpreconditioned Jacobian-free NewtonKrylov solver. A tight nonlinear tolerance is required to achieve exact energy conservation. Crucial to our implementation is the concept of particle enslavement, which segregates the particle orbit integration procedure in the evaluation of the nonlinear residual, and thus allows a completely general treatment of the particle orbits. This flexibility has been exploited 
in this study to implement an adaptive charge-conserving particle mover, which is compatible with the exact discrete energy conservation theorem. Since particle quantities are not dependent variables, the JFNK solver features very modest memory requirements, comparable to those of fluid simulations (albeit one still needs to store old-time and new-time particle positions and velocities as auxiliary variables for the residual computation). Furthermore, being a stand-alone process, the particle orbit integration stage opens the opportunity for significant acceleration using dedicated hardware such as GPGPUs.

Numerical experiments with a multiscale problem (the ion acoustic wave) have demonstrated the superior accuracy properties of the approach. In particular, we have demonstrated that large implicit time steps can be used without accuracy degradation, and that explicit approaches need to use time steps much smaller than the CFL to provide compara-

ble accuracy. Efficiency-wise, we have argued for the potential to achieve large CPU gains of implicit methods vs. explicit ones, particularly in multidimensional applications. We have demonstrated moderate gains (up to 15) with our unpreconditioned Newton-Krylov implementation in 1D.

Albeit admittedly at a proof-of-principle level, this study has resolved a number of limitations of earlier implicit PIC studies, and has uncovered the potential of fully implicit methods for both accurate and efficient kinetic plasma simulation. Future work will focus on extending our approach to a fully electromagnetic implementation, on exploring fully coupled moment-PIC representations that are better suited for the development of preconditioning strategies, and on exploiting heterogeneous architectures (e.g., GPGPUs) for the integration of particle orbits.

\section{Acknowledgments}

The authors would like to acknowledge useful conversations with D. del-Castillo-Negrete and R. Sánchez. L. C. would like to acknowledge useful conversations with D. A. Knoll, J. N. Shadid, and J. U. Brackbill, and the early contributions by G. Lapenta and H. J. Kim while Kim was a summer student at Los Alamos National Laboratory. This work has been funded by the Oak Ridge National Laboratory (ORNL) Directed Research and Development program (LDRD). ORNL is operated by UT-Battelle for the US Department of Energy under contract DE-AC05-00OR22725. 


\section{Appendix}

\section{Appendix A. Derivation of exact charge-conserving 1D particle mover}

We proceed to provide a simple derivation of the 1D charge-conserving scheme employed in this work, which differs from others reported in the literature as explained in the main text. As in the main text, we define the current and charge density $j$ and $\rho$ as

$$
\begin{aligned}
j_{i} & =\sum_{p} q_{p} v_{p} S_{m-1}\left(x_{p}-x_{i}\right) / \Delta x \\
\rho_{i+1 / 2} & =\sum_{p} q_{p} S_{m}\left(x_{p}-x_{i+1 / 2}\right) / \Delta x
\end{aligned}
$$

where $q_{p}$ is the particle charge, $v_{p}$ is the particle velocity, $S_{m}$ is shape function which is a B-spline of order $m$. Note that the shape function used for the current density is one order lower than that for the charge density. In the limit of $\Delta t \rightarrow 0$, it is straightforward to show that

$$
\begin{aligned}
\frac{\partial \rho_{i+1 / 2}}{\partial t} & =\sum_{p} \frac{q_{p}}{\Delta x} \frac{\partial S_{m}\left(x_{p}-x_{i+1 / 2}\right)}{\partial t} \\
& =\sum_{p} \frac{q_{p}}{\Delta x} \frac{\partial S_{m}}{\partial x_{p}} \frac{\partial x_{p}}{\partial t} \\
& =\sum_{p} \frac{q_{p}}{\Delta x} \frac{S_{m-1}\left(x_{p}-x_{i}\right)-S_{m-1}\left(x_{p}-x_{i+1}\right)}{\Delta x} v_{p} \\
& =-\frac{j_{i+1}-j_{i}}{\Delta x}
\end{aligned}
$$

where $\partial_{x_{p}} S_{m}\left(x_{p}-x_{i+1 / 2}\right)=-\partial_{x_{i}} S_{m}\left(x_{p}-x_{i+1 / 2}\right)=\left(S_{m-1}\left(x_{p}-x_{i}\right)-S_{m-1}\left(x_{p}-x_{i+1}\right)\right) / \Delta x$ is used. With a Crank-Nicolson discretization in time we find that:

$$
\begin{aligned}
\frac{\rho_{i}^{n+1}-\rho_{i}^{n}}{\Delta t} & =\sum_{p} \frac{q_{p}}{\Delta x} \frac{S_{m}\left(x_{p}^{n+1}-x_{i}\right)-S_{m}\left(x_{p}^{n}-x_{i}\right)}{\Delta t} \\
& =\sum_{p} \frac{q_{p}}{\Delta x} \frac{\frac{\partial S_{m}\left(x_{p}^{n+1 / 2}-x_{i}\right)}{\partial x_{p}}\left(x_{p}^{n+1}-x_{p}^{n}\right)}{\Delta t} \\
& =\sum_{p} \frac{q_{p}}{\Delta x} v_{p}^{n+1 / 2} \frac{S_{m-1}\left(x_{p}^{n+1 / 2}-x_{i}\right)-S_{m-1}\left(x_{p}^{n+1 / 2}-x_{i+1}\right)}{\Delta x} \\
& =-\frac{j_{i+1}^{n+1 / 2}-j_{i}^{n+1 / 2}}{\Delta x},
\end{aligned}
$$

where we have Taylor expanded $S_{m}\left(x_{p}^{n+1}-x_{i}\right)$ and $S_{m}\left(x_{p}^{n}-x_{i}\right)$ about $x_{p}^{n+1 / 2}$.

The exact charge conservation is valid only when the particle is moving within a cell and $m \leq 2$, because the 2 nd-order derivative terms cancel exactly, and no higher-order terms are 
present in the expansions. This is seen as follows.

$$
\begin{aligned}
S_{m}\left(x_{p}^{n+1}-x_{i}\right)= & S_{m}\left(x_{p}^{n+1 / 2}-x_{i}\right)+\frac{\partial S_{m}}{\partial x_{p}}\left(x_{p}^{n+1}-x_{p}^{n+1 / 2}\right)+ \\
& +\frac{\partial^{2} S_{m}}{\partial x_{p}^{2}} \frac{\left(x_{p}^{n+1}-x_{p}^{n+1 / 2}\right)^{2}}{2} \\
S_{m}\left(x_{p}^{n}-x_{i}\right)= & S_{m}\left(x_{p}^{n+1 / 2}-x_{i}\right)+\frac{\partial S_{m}}{\partial x_{p}}\left(x_{p}^{n}-x_{p}^{n+1 / 2}\right)+ \\
& +\frac{\partial^{2} S_{m}}{\partial x_{p}^{2}} \frac{\left(x_{p}^{n}-x_{p}^{n+1 / 2}\right)^{2}}{2}
\end{aligned}
$$

Subtracting Eq.A.4 from Eq. A.3, we find exactly that:

$$
S_{m}\left(x_{p}^{n+1}-x_{i}\right)-S_{m}\left(x_{p}^{n}-x_{i}\right)=\frac{\partial S_{m}}{\partial x}\left(x_{p}^{n+1}-x_{p}^{n}\right),
$$

which has been used in the derivation above. Since the second derivative of the B-spline is piecewise continuous only within a cell, it is required that particles stop at cell boundaries.

\section{Appendix B. Local error estimate of particle orbit integrator}

We proceed to outline the derivation of the local error estimate for the particle orbit equations, required for Eq. (19) in the main text. In what follows, we omit the subscript "p" in particle quantities for convenience. Let us consider the initial value problem for the particle orbits:

$$
\begin{aligned}
& \frac{\partial x}{\partial t}=v(t, x), x(t=0)=x^{0} \\
& \frac{\partial v}{\partial t}=a(t, x), v(t=0)=v^{0} .
\end{aligned}
$$

A forward Euler's temporal discretization method gives:

$$
\begin{aligned}
& x_{E}^{\nu+1}=x^{\nu}+v\left(t^{\nu}, x^{\nu}\right) \Delta \tau \\
& v_{E}^{\nu+1}=v^{\nu}+a\left(t^{\nu}, x^{\nu}\right) \Delta \tau .
\end{aligned}
$$

Using the forward Euler's method as the predictor in a second-order, time-centered discretization gives:

$$
\begin{aligned}
& x_{H}^{\nu+1}=x^{\nu}+\frac{\Delta \tau}{2}\left[v\left(t^{\nu}, x^{\nu}\right)+v\left(t^{\nu+1}, x_{E}^{\nu+1}\right)\right], \\
& v_{H}^{\nu+1}=v^{\nu}+\frac{\Delta \tau}{2}\left[a\left(t^{\nu}, x^{\nu}\right)+a\left(t^{\nu+1}, x_{E}^{\nu+1}\right)\right],
\end{aligned}
$$

which is the so-called Heun's formula. A Taylor expansion readily shows that forward Euler is first-order accurate, while Heun's formula is second-order accurate:

$$
\begin{aligned}
& x_{E}^{\nu+1}=x^{\nu}+\Delta \tau v\left(t^{\nu}, x^{\nu}\right)+O\left(\Delta \tau^{2}\right), \\
& x_{H}^{\nu+1}=x^{\nu}+\frac{\Delta \tau}{2}\left[v\left(t^{\nu}, x^{\nu}\right)+v\left(t^{\nu+1}, x_{E}^{\nu+1}\right)\right]+O\left(\Delta \tau^{3}\right),
\end{aligned}
$$


The local error introduced in the position by the Euler step can be readily estimated by subtracting these two expansions, to find [41]:

$$
l e_{x}=\left[v\left(t^{\nu+1}, x_{E}^{\nu+1}\right)-v\left(t^{\nu}, x^{\nu}\right)\right] \Delta \tau / 2+O\left(\Delta \tau^{3}\right) \approx \frac{\Delta \tau^{2}}{2} a\left(t^{\nu}, x^{\nu}\right)
$$

where we have used Eq. B.2 for the last step. Similarly, we find the following error estimate for the velocity:

$$
l e_{v}=\left[a\left(t^{\nu+1}, x_{E}^{\nu+1}\right)-a\left(t^{\nu}, x^{\nu}\right)\right] \Delta \tau / 2+O\left(\Delta \tau^{3}\right) \approx \frac{\Delta \tau^{2}}{2}\left(\frac{\partial a}{\partial x} v\right)^{\nu}
$$

where we have Taylor-expanded the acceleration in the last step. Equations (B.5) and (B.6) are reported in the main text.

\section{References}

[1] C. K. Birdsall, A. B. Langdon, Plasma Physics via Computer Simulation, McGraw-Hill, New York, 2005.

[2] R. W. Hockney, J. W. Eastwood, Computer Simulation Using Particles, Taylor \& Francis, Inc, Bristol, UK, 1988.

[3] Y. N. Grigoryev, V. A. Vshivkov, M. P. Fedoruk, Numerical Particle-in-cell Methods: Theory and Applications, Walter de Gruyter Inc, Boston, 2002.

[4] J. M. Dawson, Plasma oscillations of a large number of electron beams, Phys. Rev. 118 (2) (1960) 381-389.

[5] K. J. Bowers, B. J. Albright, L. Yin, B. Bergen, T. J. T. Kwan, Ultrahigh performance three-dimensional electromagnetic relativistic kinetic plasma simulation, Phys. Plasmas $15(5)$.

[6] R. J. Mason, Implicit moment particle simulation of plasmas, J. Comput. Phys. 41 (2) (1981) $233-44$.

[7] J. Denavit, Time-filtering particle simulations with $\omega_{p e} \Delta t \gg 1$, J. Comput. Phys. 42 (2) (1981) $337-66$.

[8] J. U. Brackbill, D. W. Forslund, An implicit method for electromagnetic plasma simulation in two dimensions, J. Comput. Phys. 46 (1982) 271.

[9] H. X. Vu, J. U. Brackbill, CELEST1D: an implicit, fully kinetic model for low-frequency, electromagnetic plasma simulation, Comput. Phys. Commun. 69 (1992) 253-276.

[10] A. Friedman, A. B. Langdon, B. I. Cohen, A direct method for implicit particle-in-cell simulation, Comm. Plasma Phys. Controlled Fusion 6 (6) (1981) 225 - 36.

[11] B. I. Cohen, A. B. Langdon, A. Friedman, Implicit time integration for plasma simulation, J. Comput. Phys. 46 (1) (1982) $15-38$. 
[12] A. B. Langdon, B. I. Cohen, A. Friedman, Direct implicit large time-step particle simulation of plasmas, J. Comput. Phys. 51 (1) (1983) $107-38$.

[13] D. C. Barnes, T. Kamimura, J.-N. Leboeuf, T. Tajima, Implicit particle simulation of magnetized plasmas, J. Comput. Phys. 52 (3) (1983) 480 - 502.

[14] J. U. Brackbill, D. W. Forslund, Simulation of low-frequency electromagnetic phenomena in plasmas, in: J. U. Brackbill, B. I. Cohen (Eds.), Multiple time scales, Academic Press, 1985.

[15] A. B. Langdon, D. C. Barnes, Direct implicit plasma simulation, in: J. U. Brackbill, B. I. Cohen (Eds.), Multiple time scales, Academic Press, New York, 1985, pp. 335-375.

[16] B. I. Cohen, Multiple time-scale methods in particle simulations of plasma, Particle Accelerators 19 (1986) 227-236.

[17] D. W. Hewett, A. B. Langdon, Electromagnetic direct implicit plasma simulation, J. Comput. Phys. 72 (1) (1987) $121-55$.

[18] A. Friedman, A second-order implicit particle mover with adjustable damping, J. Comput. Phys. 90 (2) (1990) 292-312.

[19] T. Kamimura, E. Montalvo, D. C. Barnes, J. N. Leboeuf, T. Tajima, Implicit particle simulation of electromagnetic plasma phenomena 100 (1) (1992) 77-90.

[20] G. Lapenta, J. U. Brackbill, CELEST3D: Implicit particle in cell simulation of space plasmas in three dimensions, in: 16th International Conference on the Numerical Simulation of Plasmas, Santa Barbara,CA, USA, 1998.

[21] B. I. Cohen, A. B. Langdon, D. W. Hewett, R. J. Procassini, Performance and optimization of direct implicit particle simulation, J. Comput. Phys. 81 (1) (1989) 151 168.

[22] C. T. Kelley, Iterative Methods for Linear and Nonlinear Equations, Society for Industrial and Applied Mathematics (SIAM), Philadelphia, 1995.

[23] H. J. Kim, L. Chacón, G. Lapenta, Fully implicit particle-in-cell algorith, in: Bull. Am. Phys. Soc., Vol. 50 (8), 2005, abstract CP1.70.

[24] G. Chen, L. Chacón, D. C. Barnes, An energy-conserving nonlinearly converged implicit particle-in-cell (PIC) algorithm, in: Bull. Am. Phys. Soc., Vol. 55 (15), 2010, abstract TP9.34.

[25] H. R. Lewis, Energy-Conserving Numerical Approximations for Vlasov Plasmas, J. Comput. Phys. 6 (1970) 136-141.

[26] J. Villasenor, O. Buneman, Rigorous charge conservation for local electromagnetic field solvers, Comput. Phys. Commun. 69 (1992) 306-316. 
[27] B. I. Cohen, R. P. Freis, V. Thomas, Orbit-averaged implicit particle codes, J. Comput. Phys. 45 (3) (1982) 345-366.

[28] A. B. Langdon, Implicit plasma simulation, Space Science Reviews 42 (1) (1985) 67-83.

[29] M. Drouin, L. Gremillet, J.-C. Adam, A. Héron, Particle-in-cell modeling of relativistic laser-plasma interaction with the adjustable-damping, direct implicit method, J. Comput. Phys. 229 (12) (2010) 4781-4812.

[30] A. B. Langdon, Analysis of the time integration in plasma simulation, J. Comput. Phys. 30 (2) (1979) $202-221$.

[31] O. Buneman, Fast numerical procedures for computer experiments on relativistic plasmas, in: Relativistic Plasmas, 1968, pp. 205-219.

[32] R. L. Morse, C. W. Nielson, Numerical simulation of the Weibel instability in one and two dimensions, Phys. Fluids 14 (1971) 830.

[33] T. Z. H. Esirkepov, Exact charge conservation scheme for Particle-in-Cell simulation with an arbitrary form-factor, Comput. Phys. Commun. 135 (2) (2001) 144-153.

[34] C. K. Birdsall, N. Maron, Plasma self-heating and saturation due to numerical instabilities, J. Comput. Phys. 36 (1) (1980) 1-19.

[35] L. Chacón, D. C. Barnes, D. A. Knoll, G. H. Miley, An implicit energy-conservative 2D Fokker-Planck algorithm: Ii-jacobian-free Newton-Krylov solver, J. Comput. Phys. 157 (2000) 654-682.

[36] R. S. Dembo, S. C. Eisenstat, T. Steihaug, Inexact Newton methods, SIAM J. Numer. Anal. 19 (2) (1982) 400.

[37] T. Stix, Waves in plasmas, Amer Inst of Physics, New York, 1992.

[38] L. Chacón, D. A. Knoll, J. M. Finn, Implicit, nonlinear reduced resistive MHD nonlinear solver, J. Comput. Phys. 178 (1) (2002) 15-36.

[39] L. Chacón, D. A. Knoll, A 2D high- $\beta$ Hall MHD implicit nonlinear solver, J. Comput. Phys. 188 (2) (2003) 573-592.

[40] L. Chacón, An optimal, parallel, fully implicit Newton-Krylov solver for threedimensional visco-resistive magnetohydrodynamics, Phys. Plasmas 15 (2008) 056103.

[41] L. F. Shampine, Stiffness and nonstiff differential equation solvers, II: detecting stiffness with Runge-Kutta methods, ACM Trans. Math. Softw. 3 (1) (1977) 44-53. 